\title{
The cosmological bootstrap: weight-shifting operators and scalar seeds
}

\section{Daniel Baumann, ${ }^{a}$ Carlos Duaso Pueyo, ${ }^{a}$ Austin Joyce, ${ }^{b}$ Hayden Lee ${ }^{c}$ and Guilherme L. Pimentel ${ }^{a, d}$}

\author{
${ }^{a}$ Institute of Physics, University of Amsterdam, \\ Amsterdam, $1098 \mathrm{XH}$, The Netherlands \\ ${ }^{b}$ Department of Physics, Columbia University, \\ New York, NY 10027, U.S.A. \\ ${ }^{c}$ Department of Physics, Harvard University, \\ Cambridge, MA 02138, U.S.A. \\ ${ }^{d}$ Lorentz Institute for Theoretical Physics, \\ Leiden, 2333 CA, The Netherlands
}

E-mail: dbaumann@uva.nl, c.duasopueyo@uva.nl, a.p.joyce@uva.nl, hylee@g.harvard.edu, G.LeitePimentel@uva.nl

ABSTRACT: A key insight of the bootstrap approach to cosmological correlations is the fact that all correlators of slow-roll inflation can be reduced to a unique building block - the four-point function of conformally coupled scalars, arising from the exchange of a massive scalar. Correlators corresponding to the exchange of particles with spin are then obtained by applying a spin-raising operator to the scalar-exchange solution. Similarly, the correlators of massless external fields can be derived by acting with a suitable weightraising operator. In this paper, we present a systematic and highly streamlined derivation of these operators (and their generalizations) using tools of conformal field theory. Our results greatly simplify the theoretical foundations of the cosmological bootstrap program.

Keywords: Conformal Field Theory, Cosmology of Theories beyond the SM, Models of Quantum Gravity, Space-Time Symmetries

ArXiv EPrint: 1910.14051 


\section{Contents}

1 Introduction $\quad 2$

2 De Sitter four-point functions $\quad 4$

2.1 Boundary correlators 4

2.2 Scalar seed functions

2.3 Spin-exchange solutions 9

2.4 Inflationary correlators 9

3 CFTs in embedding space $\quad 9$

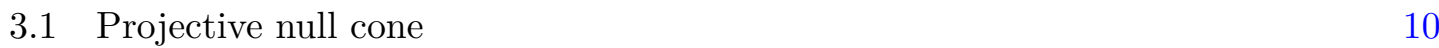

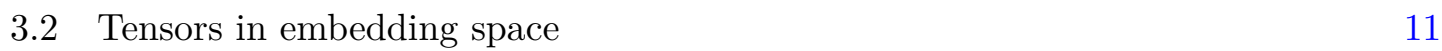

$\begin{array}{lll}3.3 & \text { Conformal correlators } & 12\end{array}$

4 Exchange of spinning particles $\quad 13$

4.1 Spin-raising operator 14

$\begin{array}{ll}4.2 & \text { Raising internal spin } \\ \end{array}$

$\begin{array}{lll}4.2 .1 & \text { Spin-1 exchange } & 14\end{array}$

$\begin{array}{lll}4.2 .2 & \text { Spin-2 exchange } & 16\end{array}$

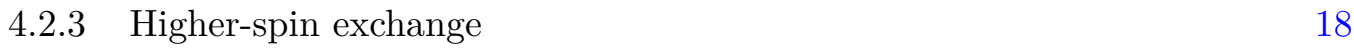

5 Inflationary three-point functions $\quad 19$

$\begin{array}{ll}5.1 \text { Weight-raising operator } & 19\end{array}$

5.2 Raising external weight 20

5.3 From de Sitter to inflation 21

5.4 Partially massless exchange 22

6 Conclusions $\quad 27$

A Weight shifting in embedding space $\quad 28$

A.1 General preliminaries 28

$\begin{array}{lll}\text { A.2 Some group theory } & 29\end{array}$

A.3 Vector representation 30

A.4 Adjoint representation 34

B Weight shifting in Fourier space $\quad 36$

$\begin{array}{lll}\text { B.1 Projection to position space } & 36\end{array}$

$\begin{array}{lll}\text { B.2 } & \text { Fourier-transformed operators } & 37\end{array}$

B.3 A few simple examples $\quad 39$

C Polarization tensors 40

D Notation and conventions $\quad 43$ 


\section{Introduction}

It is remarkable how much physics can sometimes be derived from just a few basic principles. For example, the form of all consistent particle interactions is a nearly inevitable consequence of the twin pillars of quantum mechanics and special relativity. This is made manifest in the "S-matrix bootstrap," where simple self-consistency requirements (such as Lorentz symmetry, locality and causality) completely fix the analytic structure of tree-level scattering amplitudes. Recently, a similar bootstrap philosophy was applied to cosmological correlation functions [1] (see also [2-7]). Working under the lamppost of slow-roll inflation with weak couplings to extra massive particles, this "cosmological bootstrap" allowed for a complete classification of all scalar three- and four-point functions at tree level.

The standard approach to computing inflationary correlation functions involves following the time-evolution of fields during inflation. From this viewpoint, locality is a fundamental input, and the interactions between particles lead to complicated time integrals that encode the manifestly local time evolution. The outputs of this procedure are late-time correlation functions that live on the (spacelike) future boundary of de Sitter space. These correlation functions are encoded in the statistics of late-time cosmological observables, and so form the fundamental observable output of inflation. The bootstrap philosophy is to focus on these final observable quantities and construct them directly, granting primacy to principles aside from manifest locality. In particular, the cosmological bootstrap exploits the approximate de Sitter symmetries - which act as conformal transformations on the boundary [8-12] - along with consistency requirements on the singularity structure of correlation functions to reconstruct the output of bulk time evolution without ever talking about time. Correlation functions instead arise as solutions (with particular singularities) of conformal Ward identities [1]. (See [13-35] for other studies on conformal correlators in momentum space.) Although these solutions describe static boundary correlators, they encode time-dependent processes in the bulk, including the production and decay of very massive particles $[12,36-56]$.

One of the main insights of the bootstrap approach is the fact that all correlators can be reduced to a unique building block - the de Sitter four-point function of conformally coupled scalars, mediated by the exchange of a massive scalar. Solutions corresponding to the exchange of particles with spin are obtained by applying a spin-raising operator, $\mathcal{S}$, to the scalar-exchange solution. Similarly, solutions for massless external fields are derived by acting with a set of weight-raising operators, $\mathcal{W}$. Finally, the de Sitter four-point functions lead to inflationary three-point functions when one of the external legs is evaluated on the time-dependent background (see figure 1). The derivation of these spin-raising and weight-shifting operators in [1] was somewhat unsatisfactory, involving a combination of bulk reasoning, boundary considerations, and educated guesswork. In this paper, we will present a more systematic derivation of these operators (and their generalizations) using tools of conformal field theory (CFT). This has the advantage of being purely intrinsic to the boundary, along with placing these operators in a broader context, opening up new avenues to the cosmological bootstrap [57]. 

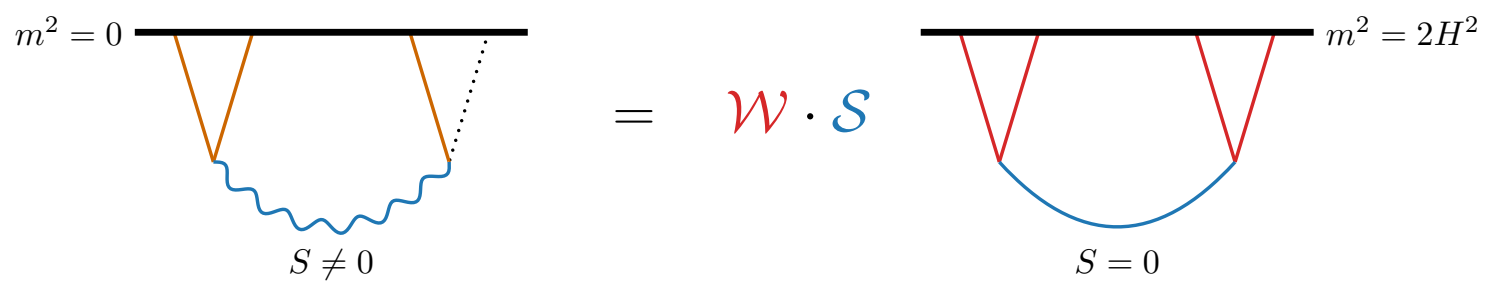

Figure 1. Schematic illustration of the spin-raising and weight-shifting procedure studied in this paper.

Given that late-time correlation functions in de Sitter space are conformally invariant, it is natural to try to connect the study of cosmological correlators to CFT techniques. However, most of our understanding of CFTs has been developed in position space, while the natural habitat of cosmological correlators is Fourier space. If standard CFT results could be translated to Fourier space, we would learn a great deal about the structure of inflationary correlators. Moreover, the momentum-space approach can also be useful in studying CFTs in Lorentzian signature [58-62]. Unfortunately, taking the direct Fourier transform of position space CFT correlators is quite nontrivial. First of all, CFT correlators are singular at coincident points and need to be renormalized before the result can be Fourier transformed. Second, even after renormalization, the explicit Fourier transforms are technically challenging. In practice, it turns out to be easier to solve the conformal Ward identities directly in momentum space. However, even this approach quickly becomes intractable for operators with spin. Fortunately, the weight-shifting approach provides a more elegant way to proceed. This formalism allows us to generate new solutions to the conformal Ward identities by acting with differential operators on an initial seed solution.

In this paper, we point out that the relevant spin-raising and weight-shifting operators used in cosmology are equivalent to similar operators used in the CFT literature [63-65]. The latter are defined most naturally in embedding space [66-68]. We show that the CFT weight-shifting operators are easily Fourier transformed, thereby bypassing the usual challenge of relating position space and Fourier space. Moreover, we show that the lift to embedding space provides an elegant way to derive and generalize the cosmological weightshifting operators found in [1]. This new viewpoint clarifies the fact that all inflationary correlators can be obtained from a unique seed function corresponding to the exchange of a scalar particle and streamlines its derivation from the boundary perspective. The weightshifting approach also makes it clear how the spins of the external fields can be raised to obtain spinning solutions to the conformal Ward identities. We will present the details in a separate publication [57], where we show that inflationary tensor correlators can also be obtained from scalar seeds.

Outline. The plan of the paper is as follows: in section 2, we recall a few relevant results of the cosmological bootstrap [1]. In particular, we present the de Sitter four-point function of conformally coupled scalars, arising from the tree-exchange of a generic scalar. This solution is the essential building block from which all other correlators will be derived via the action of suitable differential operators. In section 3 , we briefly review the embedding space 
formalism for CFTs. Experts may skip this part without loss of continuity. In section 4, we work in embedding space to derive an operator which raises the spin of the particles exchanged in the correlators introduced in section 2, and then translate this operator to Fourier space to obtain spin-exchange solutions. In section 5 , we use the formalism to derive a weight-raising operator that transforms the four-point functions of conformally coupled scalars to those of massless scalars. We then show that the soft limit of these correlators with weakly perturbed scaling dimension leads to inflationary three-point functions. This allows for a compact expression for the inflationary bispectrum coming from the exchange of massive particles. We also present new results for the exchange of (partially) massless fields of arbitrary spin. Our conclusions are presented in section 6 . The appendices contain additional technical details and derivations: in appendix A, we provide a systematic derivation of weight-shifting operators in embedding space. In appendix B, we transform these operators to Fourier space. In appendix C, we present explicit results for the polarization tensors used in the main text. Finally, appendix D collects important variables used in this work.

Notation and conventions. Unless stated otherwise, we will follow closely the notation and conventions used in [1]. Generic scalar operators (of dimension $\Delta$ ) will be denoted by $O$. We will use $\varphi$ and $\phi$ for operators with $\Delta=2$ and $\Delta=3$, respectively. When we need to refer to the corresponding bulk fields, we will use $\varphi$ and $\phi$. The bulk de Sitter coordinates are $x^{\mu}$ and the coordinates on the spatial boundary are $x^{i}$, with conjugate momentum $k^{i}$. The boundary has $d$ spatial dimensions, and we often specialize to $d=3$, corresponding to the four-dimensional de Sitter space that seems to be relevant for our universe. Our convention for the $d$-dimensional Fourier transform is

$$
O(\vec{x})=\int \frac{\mathrm{d}^{d} k}{(2 \pi)^{d}} e^{-i \vec{k} \cdot \vec{x}} O_{\vec{k}}
$$

The coordinates in the embedding space formalism are $X^{M}$, with $M=-1,0,1, \ldots, d$, and the corresponding lightcone coordinates are $X^{ \pm}=X^{0} \pm X^{-1}$.

\section{De Sitter four-point functions}

In order to make our discussion self-contained, we begin with a brief review of relevant results from [1], focusing on the bare minimum of background material required for our present purposes. All derivations and further details can be found in [1].

\subsection{Boundary correlators}

In the standard cosmological model, all cosmological correlators can be traced back to the end of inflation (or the beginning of the hot Big Bang), where they reside on the spacelike boundary of an approximate de Sitter spacetime (see figure 2). The time dependence of bulk interactions is encoded in the momentum dependence of these boundary correlators. In particular, massive particles can be produced and decay during inflation, leaving their imprint in the nontrivial correlations on the boundary. In the case of single-field slow-roll inflation with sufficiently weak couplings to additional massive particles, the inflationary correlators 


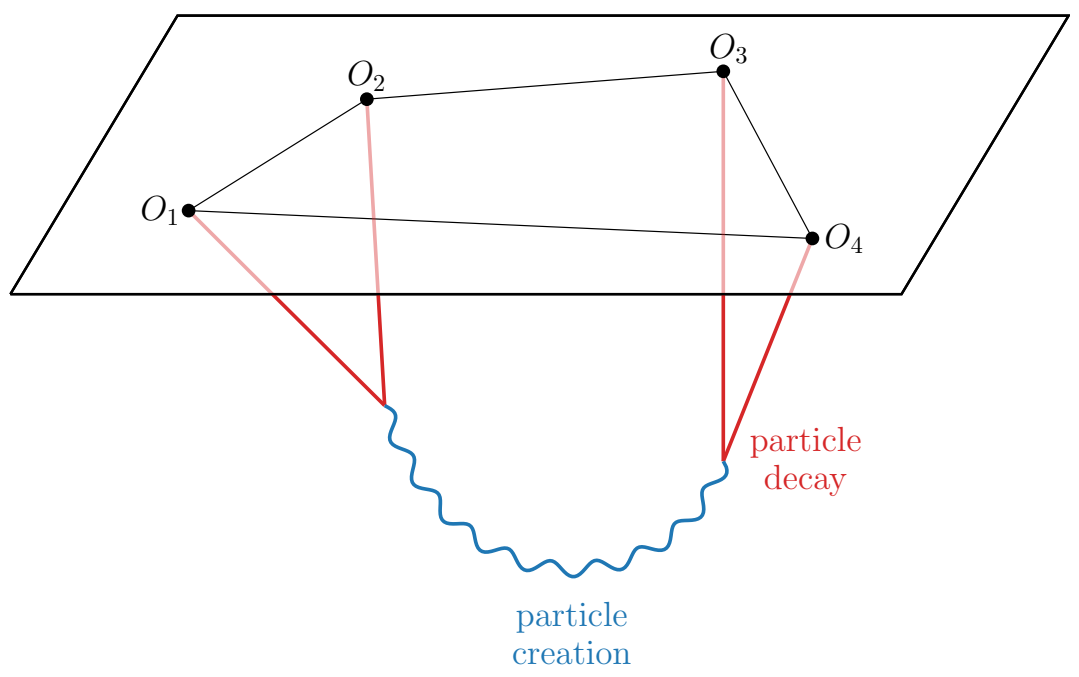

Figure 2. Illustration of particle creation and decay producing correlations on the future boundary of the de Sitter spacetime. The momentum dependence of the boundary correlators encodes the time dependence of the processes in the bulk.

are strongly constrained by the conformal symmetry that the boundary theory inherits from the isometries of the bulk quasi-de Sitter spacetime. This is a promising arena to attempt to directly construct - or bootstrap - correlators on the future boundary by exploiting the large degree of symmetry and the expected analytic properties of tree-level processes.

It is useful to briefly review how the symmetry constraints on late-time correlation functions arise in inflationary cosmology. The setting is de Sitter space in the inflationary slicing, which is described by the line element

$$
\mathrm{d} s^{2}=\frac{1}{H^{2} \eta^{2}}\left(-\mathrm{d} \eta^{2}+\mathrm{d} \vec{x}^{2}\right) .
$$

This spacetime has 10 Killing vectors associated with the following generators

$$
\begin{aligned}
P_{i} & =\partial_{i}, & D & =-\eta \partial_{\eta}-x^{i} \partial_{i}, \\
J_{i j} & =x_{i} \partial_{j}-x_{j} \partial_{i}, & K_{i} & =2 x_{i} \eta \partial_{\eta}+\left(2 x^{j} x_{i}+\left(\eta^{2}-x^{2}\right) \delta_{i}^{j}\right) \partial_{j} .
\end{aligned}
$$

These transformations include translations $\left(P_{i}\right)$ and rotations $\left(J_{i j}\right)$, which preserve the spatial slices, as well as dilations $(D)$ and special conformal transformations $\left(K_{i}\right)$. We will assume that any additional matter fields only weakly break these de Sitter symmetries. This implies that the late-time correlation functions of fields will transform like correlators in a CFT. To see how these constraints arise, let us consider the evolution of a scalar field in de Sitter space. At late times, a scalar field behaves as

$$
\sigma(\vec{x}, \eta \rightarrow 0)=\sigma_{+}(\vec{x}) \eta^{\Delta_{+}}+\sigma_{-}(\vec{x}) \eta^{\Delta_{-}} .
$$

We see that the field has two characteristic fall-offs, whose time dependence is fixed by the mass of the field, $m$, through the relation

$$
\Delta_{ \pm}=\frac{3}{2} \pm \sqrt{\frac{9}{4}-\frac{m^{2}}{H^{2}}} .
$$


From this late-time scaling, we infer that the coefficient functions in (2.3) must transform as

$$
\begin{aligned}
P_{i} \sigma_{ \pm} & =\partial_{i} \sigma_{ \pm}, & D \sigma_{ \pm} & =-\left(\Delta_{ \pm}+x^{i} \partial_{i}\right) \sigma_{ \pm} \\
J_{i j} \sigma_{ \pm} & =\left(x_{i} \partial_{j}-x_{j} \partial_{i}\right) \sigma_{ \pm}, & K_{i} \sigma_{ \pm} & =\left(2 x_{i} \Delta_{ \pm}+2 x_{i} x^{j} \partial_{j}-x^{2} \partial_{i}\right) \sigma_{ \pm}
\end{aligned}
$$

where $\Delta_{ \pm}$is the weight of the operator late-time field coefficient $\sigma_{ \pm}$. This implies that correlation functions of $\sigma_{ \pm}$- and hence late-time correlation functions of fields in de Sitter space - obey the same kinematic constraints as conformal correlators. This allows us to leverage insights from the study of CFT to learn about inflationary correlators.

We will be interested in the correlation functions of the late-time spatial profiles of fields in de Sitter space, focusing on the case of "light fields," for which $m^{2} / H^{2} \leq 9 / 4$. For these fields, the dominant fall-off at late times is given by $\Delta_{-}$, so that the physically interesting correlation functions are those of $\sigma_{-}$. However, in practice we will compute the correlation functions of $\sigma_{+}$because these take a slightly simpler form. These correlators are related to those of $\sigma_{-}$by a shadow transform, which in Fourier space amounts to a simple multiplication by factors of power spectra. ${ }^{1}$ We will denote the conformal weight of the dual boundary operators by $\Delta \equiv \Delta_{+}$. The cases of primary interest in this paper are conformally coupled scalars (with $m^{2}=2 H^{2}$ or $\Delta=2$ ) and massless scalars (with $\Delta=3$ ). Though we restrict our attention to light external fields, we will allow internal fields of arbitrary weights.

In the cosmological context we are interested in correlation functions in Fourier space, in order to take advantage of the translational invariance of the spatial hypersurfaces. We should therefore translate the constraints coming from de Sitter/conformal invariance into momentum space. To illustrate this, let us consider a four-point function of scalar operators, which in momentum space takes the form

$$
\left\langle O_{1} O_{2} O_{3} O_{4}\right\rangle=F\left(k_{1}, k_{2}, k_{3}, k_{4}, s, t\right) \times(2 \pi)^{3} \delta^{3}\left(\vec{k}_{1}+\cdots+\vec{k}_{4}\right),
$$

where $O_{n} \equiv O_{n}\left(\vec{k}_{n}\right)$ are generic operators of scaling dimensions $\Delta_{n}$. Invariance under spatial rotations and translations implies that the four-point function $F$ is a function of six independent variables, which we take to be $k_{n} \equiv\left|\vec{k}_{n}\right|, s \equiv\left|\vec{k}_{1}+\vec{k}_{2}\right|$ and $t \equiv\left|\vec{k}_{2}+\vec{k}_{3}\right|$. To be invariant under dilations (D) and special conformal transformations (SCTs), the function $F$ must satisfy the following conformal Ward identities

$$
\begin{aligned}
\mathrm{D}: \quad 0 & =\left[9-\sum_{n=1}^{4}\left(\Delta_{n}-k_{n}^{j} \frac{\partial}{\partial k_{n}^{j}}\right)\right] F, \\
\mathrm{SCT}: \quad 0 & =\sum_{n=1}^{4}\left[\left(\Delta_{n}-3\right) \frac{\partial}{\partial k_{n, i}}-k_{n}^{j} \frac{\partial^{2}}{\partial k_{n}^{j} k_{n, i}}+\frac{k_{n}^{i}}{2} \frac{\partial^{2}}{\partial k_{n}^{j} k_{n, j}}\right] F,
\end{aligned}
$$

which are the momentum-space equivalents of the constraints in (2.5). To satisfy (2.7) it is sufficient to write

$$
F=s^{\Delta_{t}-9} \hat{F},
$$

\footnotetext{
${ }^{1}$ Conformal primary operators of the same spin that are related by $\tilde{\Delta}=d-\Delta$ are so-called shadows of each other. These operators generate equivalent representations of the conformal group and can be mapped to each other by means of the shadow transform. For scalar operators in momentum space, the shadow transform is implemented by the map $O_{\tilde{\Delta}}(\vec{k})=\left\langle O_{\tilde{\Delta}}(\vec{k}) O_{\tilde{\Delta}}(-\vec{k})\right\rangle O_{\Delta}(\vec{k})$. See appendix A of [72] for more details.
} 
where $\Delta_{t} \equiv \sum_{n} \Delta_{n}$ and $\hat{F}$ is a dimensionless function. The form of $\hat{F}$ will be determined by the remaining Ward identity (2.8) and the singularities of tree-level processes.

Once we have understood that cosmological correlation functions obey these symmetry constraints, we can cast aside the bulk interpretation of these correlators as arising from local causal and unitary time evolution, and attempt to reconstruct the corresponding correlation functions by directly solving (2.8). This is the approach that we will take in this paper: the goal is to provide a systematic purely boundary derivation and interpretation of correlation functions corresponding to bulk tree-level exchange of massive particles. This is both practically useful - it will allow us to characterize slow-roll inflationary three-point functions in a completely universal way - as well as conceptually interesting, as it provides insight into how bulk time evolution is encoded in the (static) boundary correlation functions.

\subsection{Scalar seed functions}

As we alluded to before, a case of special interest is the four-point function of the operator dual to conformally coupled scalars $\varphi$ (with $\Delta=2$ ), mediated by the tree-exchange of massive scalars $\sigma$. In this case the kinematics further simplifies, and the four-point function can be written as a function of only two kinematic variables. In particular, the $s$-channel contribution takes the form

$$
F=s^{-1} \hat{F}(u, v), \quad \text { where } \quad \begin{aligned}
u & \equiv \frac{s}{k_{1}+k_{2}}, \\
v & \equiv \frac{s}{k_{3}+k_{4}} .
\end{aligned}
$$

The ansatz (2.10) automatically satisfies two of the three equations contained in (2.8). After changing momentum variables, the remaining constraint equation can be written as [1]

$$
\left(\Delta_{u}-\Delta_{v}\right) \hat{F}=0
$$

where $\Delta_{u} \equiv u^{2}\left(1-u^{2}\right) \partial_{u}^{2}-2 u^{3} \partial_{u}$. In general these equations have many solutions, but tree-level bulk physics is captured by solutions with a particular singularity structure.

In [1], the solutions of (2.11) were classified, for both contact interactions and tree-level exchange of massive particles. A large class of contact solutions is extremely simple, and can be written as $[12]$

$$
\hat{C}_{n}=\Delta_{u}^{n} \hat{C}_{0}, \quad \text { where } \quad \hat{C}_{0}=\frac{u v}{u+v} .
$$

The seed contact solution $\hat{C}_{0}$ arises from a $\varphi^{4}$ interaction in the bulk. Repeated application of the operator $\Delta_{u}$ produces the additional solutions $\hat{C}_{n}$ corresponding to higher-derivative interactions in the bulk. ${ }^{2}$

\footnotetext{
${ }^{2}$ An important caveat is that this is not the most general possible contact solution. The contact solutions shown are the ones that come from integrating out scalar particles at tree level. Integrating out higherspin particles can produce contact solutions with dependence on additional kinematic invariants. These additional contact solutions can be generated by acting with the weight-shifting operators introduced in section 4.2 on the contact solutions in (2.12).
} 
In the case of tree exchange, the partial differential equation (2.11) can be written as two ordinary differential equations in $u$ and $v$ separately:

$$
\begin{aligned}
& \left(\Delta_{u}+M^{2}\right) \hat{F}=\hat{C}_{n}, \\
& \left(\Delta_{v}+M^{2}\right) \hat{F}=\hat{C}_{n},
\end{aligned}
$$

where $\hat{C}_{n}$ is one of the contact solutions in $(2.12)$ and $M^{2} \equiv\left(1-\Delta_{\sigma}\right)\left(\Delta_{\sigma}-2\right)$, which can be related to the mass of the exchanged particle via (2.4). ${ }^{3}$ The replacement of the PDE in (2.11) with two ODEs in (2.13) can be understood as a manifestation of locality at tree level. In particular, these differential equations can be derived from the bulk perspective using the equation of motion that the Green's function for the exchanged field obeys [1].

The equations (2.13) are second-order ordinary differential equations, so they require two boundary conditions. It is natural to impose boundary conditions at the singular points of the differential equation, $u=0, \pm 1$. It is easy to see that a generic solution has the following logarithmic singularities

$$
\begin{aligned}
& \lim _{u \rightarrow+1} \hat{F} \propto \log (1-u), \\
& \lim _{u, v \rightarrow-1} \hat{F} \propto \log (1+u) \log (1+v) .
\end{aligned}
$$

The limit $u \rightarrow 1$ corresponds to a collinear configuration where the momenta $\vec{k}_{1}$ and $\vec{k}_{2}$ align. In the standard Bunch-Davies vacuum, this limit should be regular. We therefore impose the absence of the singularity at $u=1$ as one boundary condition. The limit $u, v \rightarrow-1$ cannot be reached for real momenta, but corresponds to an analytic continuation in the complex plane. In this limit, the four-point function factorizes into a product of three-particle amplitudes. The correct normalization of this factorization channel (which depends on whether we are computing the wavefunction or the correlator) provides a second boundary condition. These two boundary conditions uniquely fix the solutions to (2.13) given the form of the contact solutions $\hat{C}_{n}$.

The final singularity as $u \rightarrow 0$ is physically the most interesting; in this limit the correlation function displays a characteristic non-analyticity

$$
\lim _{u \rightarrow 0} \hat{F} \propto u^{\frac{1}{2} \pm i \mu}, \quad \mu \equiv \sqrt{\frac{m^{2}}{H^{2}}-\frac{9}{4}},
$$

where the parameter $\mu$ is set by the mass $m$ of the exchanged particle. The limit $u \rightarrow 0$ is the so-called collapsed limit - where two of the momenta nearly add to zero - and the characteristic ringing as we approach this limit is imprinted in inflationary three-point functions, providing a sharp way to test for the presence of these heavy states in observables. The detailed form of the solutions $\hat{F}$ can be found in [1], but won't be needed in our analysis below.

\footnotetext{
${ }^{3}$ Explicitly, we have $M^{2}=m^{2} / H^{2}-2$, where $m$ is the mass of the exchanged field in the bulk. The coupling to gravity is such that a massless scalar corresponds to $m^{2}=0$. Note that, in the limit $M \rightarrow \infty$, equation (2.13) has a formal solution as a series of contact terms of the form (2.12), which is the EFT expansion arising from integrating out a heavy particle.
} 


\section{$2.3 \quad$ Spin-exchange solutions}

For scalar exchange, the correlators only depended on the variables $u, v$ (along with an overall power of $s$ ), but when we consider the exchange of spinning particles, the kinematics are more complicated. The four-point function will depend on the following additional variables

$$
\hat{\alpha} \equiv \frac{k_{1}-k_{2}}{s}, \quad \hat{\beta} \equiv \frac{k_{3}-k_{4}}{s}, \quad \hat{\tau} \equiv \frac{\left(\vec{k}_{1}-\vec{k}_{2}\right) \cdot\left(\vec{k}_{3}-\vec{k}_{4}\right)}{s^{2}}
$$

To determine the spin-exchange solutions, one approach is to re-solve (2.8), allowing for an additional dependence of $\hat{F}$ on $\hat{\alpha}, \hat{\beta}$ and $\hat{\tau}$. This quickly becomes very complicated and cumbersome. Fortunately, it isn't necessary to solve the Ward identities for every case separately. Instead, all spin-exchange solutions can be generated by acting with spin-raising operators on the scalar-exchange solutions. Written as a sum over helicity contributions, the spin- $S$ exchange solutions take the following form [1]

$$
\hat{F}^{(S)}=\sum_{m=0}^{S} \Pi_{S, m} \mathcal{D}_{u v}^{(S, m)} \hat{F}^{(0)}
$$

where we have defined $\hat{F}^{(0)} \equiv \hat{F}(u, v)$. In this paper, we use a group-theoretic approach inspired by known tools of conformal field theory [63, 64, 66-68] to provide a new and more elegant derivation of the relevant spin-raising operators $\mathcal{D}_{u v}^{(S, m)}$ and polarization sums $\Pi_{S, m}$ (see section 4).

\subsection{Inflationary correlators}

Our real interest for applications to inflation is in massless external fields $\phi$ (with $\Delta=3$ ), and not conformally coupled fields $\varphi$ (with $\Delta=2$ ). In [1], the dimensions/weights of the external fields were raised by acting with suitable differential operators on the solutions for conformally coupled scalars. While this got the job done, the treatment in [1] was unsatisfactory in a number of ways: $i$ ) different weight-shifting operators had to be found for each spin-exchange solution separately and $i i)$ their derivation wasn't very systematic, so that explicit results were only shown up to spin two. In this paper, we will provide a much simpler and more unified derivation of a single weight-raising operator (see section 5 ). When this operator acts on a general spin-exchange solution (2.18), it straightforwardly reproduces the results in [1] for low spins and automatically generalizes them to arbitrary spin. Weakly perturbing the scaling dimension appearing in the weight-shifting operator, $\Delta_{4}=2 \rightarrow \Delta_{4}=2-\epsilon$, where $\epsilon$ is the slow-roll parameter, and taking the soft limit $k_{4} \rightarrow 0$ provides an elegant way to obtain inflationary three-point functions for arbitrary spin exchange. The source functions appearing in these inflationary correlators will be given explicitly for any mass and spin of the exchange field.

\section{CFTs in embedding space}

We are interested in exploring the consequences of conformal symmetry on inflationary correlators, so it is useful to introduce some technical machinery to deal with conformal 


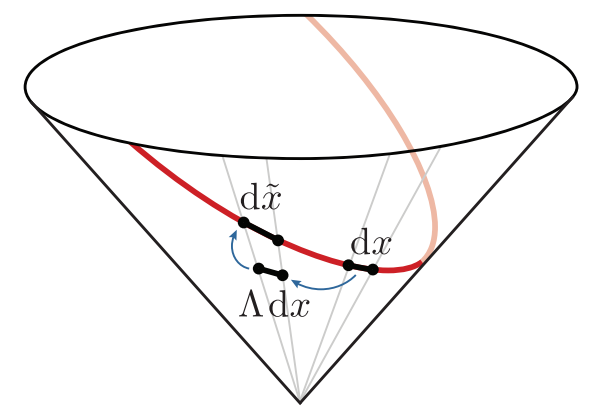

Figure 3. Illustration of the action of Lorentz transformations (and re-scalings) on points living on the Euclidean section of the lightcone in embedding space.

symmetry in a simple way. Conformal transformations are complicated nonlinear transformations and become particularly involved for spinning operators. At the same time, it is easy to show that the conformal algebra on $\mathbb{R}^{d}$ is isomorphic to the algebra of Lorentz transformations on $\mathbb{R}^{1, d+1}$. This suggests that a suitable embedding of $\mathbb{R}^{d}$ into $\mathbb{R}^{1, d+1}$, should make conformal transformations as simple as Lorentz transformations. The embedding space formalism of conformal field theory goes back to Dirac [66], and has found many powerful applications in the recent CFT literature, e.g. [63, 64]. As we will see, the formalism is particularly well-suited to describe fields with spin.

In this section, we provide a pedagogical review of CFTs in embedding space, based mostly on the excellent treatment in [69]. Experts may skip directly to section 4, where we apply this formalism to derive the weight-shifting operators of interest in cosmology.

\subsection{Projective null cone}

We begin by describing the embedding of $d$-dimensional Euclidean space as a slice through a higher-dimensional lightcone. Consider $d+2$ dimensional Minkowski space, with coordinates

$$
X^{M}, M=-1,0,1, \ldots, d,
$$

where Lorentz transformations act as

$$
X^{M} \rightarrow \Lambda^{M}{ }_{N} X^{N} .
$$

The goal is to find an embedding of $\mathbb{R}^{d}$ into $\mathbb{R}^{1, d+1}$ on which these Lorentz transformations become conformal transformations. We first restrict ourselves to points living on the null cone in the embedding space:

$$
X^{2}=0
$$

This condition is Lorentz invariant and removes one of the coordinates in (3.1). To remove a second coordinate and obtain a $d$-dimensional subspace, we define a section of the lightcone $X^{+}=f\left(X^{i}\right)$, where $X^{ \pm} \equiv X^{0} \pm X^{-1}$ are lightcone coordinates and the coordinates $X^{i}$ are identified with the coordinates $x^{i}$ on $\mathbb{R}^{d}$.

We would like to understand how Lorentz transformations act on points living on the section. In particular, we want to determine for which choice of embedding function $f\left(X^{i}\right)$ 
these transformations become conformal transformations. The action of the Lorentz group on the section is illustrated in figure 3. Each point on the section defines a lightray by connecting the point to the origin. Let $\mathrm{d} x$ be the infinitesimal interval between two nearby points on the section. The induced metric on the section relates this to the interval $\mathrm{d} s^{2}$. Since the Lorentz transformation (3.2) is an isometry, it will not change $\mathrm{d} s^{2}$. However, by itself, the Lorentz transformation will move the interval off the section. To map it back onto the section, we need to perform an additional rescaling

$$
X^{M} \rightarrow \lambda(X) X^{M}
$$

Under the combined action of (3.2) and (3.4), the induced metric on the section transforms as [69]

$$
\mathrm{d} s^{2} \rightarrow \Omega^{2}(x) \mathrm{d} s^{2}, \quad \text { with } \quad \Omega(x)=\lambda(X) .
$$

This corresponds to a conformal transformation on $\mathbb{R}^{d}$ if $\mathrm{d} s^{2}$ is flat. One can show that the latter requirement implies $f\left(X^{i}\right)=$ const. Without loss of generality, we can choose $X^{+}=1$, so that the Euclidean section of the lightcone is

$$
X^{M}=\left(X^{+}, X^{-}, X^{i}\right)=\left(1, x^{2}, x^{i}\right) .
$$

As we will show next, correlators in the $d$-dimensional Euclidean space are lifted to homogeneous functions on the lightcone of the $(d+2)$-dimensional Minkowski spacetime, where the conformal group acts as the Lorentz group.

\subsection{Tensors in embedding space}

Consider a symmetric, traceless and transverse tensor $O_{M_{1} \ldots M_{S}}$ defined on the cone $X^{2}=0$. Under the rescaling $X \rightarrow \lambda X$, the tensor transforms as

$$
O_{M_{1} \ldots M_{S}}(\lambda X)=\lambda^{-\Delta} O_{M_{1} \ldots M_{S}}(X),
$$

i.e. it is a homogeneous function of degree $\Delta$. This implies that the tensor is known everywhere on the cone if it is known on the section (3.6). The corresponding tensor on $\mathbb{R}^{d}$ is then defined through the following projection

$$
O_{i_{1} i_{2} \ldots}(x)=O_{M_{1} M_{2} \ldots}(X) \frac{\partial X^{M_{1}}}{\partial x^{i_{1}}} \frac{\partial X^{M_{2}}}{\partial x^{i_{2}}} \cdots, \quad \frac{\partial X^{M}}{\partial x^{i}}=\left(0,2 x_{i}, \delta_{i}^{j}\right) .
$$

It is straightforward to show that the scaling transformation (3.7) for $O_{M_{1} M_{2} \ldots}(X)$ implies a conformal transformation for $O_{i_{1} i_{2} \ldots}(x)$.

Contracting the tensors with auxiliary null polarization vectors $z^{i}$ and $Z^{M}$, we can write them in index-free notation

$$
\begin{aligned}
O^{(S)}(x, z) & =O_{i_{1} \cdots i_{S}}(x) z^{i_{1}} \cdots z^{i_{S}} \\
O^{(S)}(X, Z) & =O_{M_{1} \cdots M_{S}}(X) Z^{M_{1}} \cdots Z^{M_{S}} .
\end{aligned}
$$

In embedding space, any symmetric traceless tensor operator can therefore be written as a homogeneous function $O^{(S)}(X, Z)$ of the coordinates $X, Z \in \mathbb{R}^{d+1,1}$ such that $X^{2}=X \cdot Z=$ 
$Z^{2}=0$, with "gauge invariance" under $Z \rightarrow Z+\beta X$. Together with the scaling (3.7), this gauge invariance removes exactly two components per index from the tensor in embedding space, so its independent components match with those of the tensor on the Euclidean section. Under rescalings of the embedding coordinates, we have

$$
O^{(S)}(\lambda X, \alpha Z)=\lambda^{-\Delta} \alpha^{S} O^{(S)}(X, Z),
$$

where $\Delta, S$ are the dimension and spin of $O^{(S)}$. Collectively, we refer to $[\Delta, S]$ as the weight of the operator. Conformal correlators in embedding space are simply the most general Lorentz-invariant expressions with the correct scaling behavior. The projection of these functions onto the Euclidean section defines the space of conformally-invariant correlation functions on $\mathbb{R}^{d}$.

\subsection{Conformal correlators}

To illustrate the power of the embedding space formalism, we present a few examples of conformal correlators.

Consider first a set of scalar primary operators $O_{a} \equiv O_{a}\left(X_{a}\right)$, of dimension $\Delta_{a}$. Correlators of $O_{a}$ can only depend on the Lorentz-invariant inner products

$$
X_{a b} \equiv X_{a} \cdot X_{b},
$$

since $X_{a}^{2}=0$ on the lightcone. The scaling in (3.11) then uniquely fixes the two- and three-point functions of the operators to be

$$
\begin{aligned}
\left\langle O_{1} O_{2}\right\rangle & =\frac{1}{X_{12}^{\Delta_{1}}} \delta_{\Delta_{1} \Delta_{2}}, \\
\left\langle O_{1} O_{2} O_{3}\right\rangle & =\frac{c_{123}}{X_{12}^{\left(\Delta_{1}+\Delta_{2}-\Delta_{3}\right) / 2} X_{23}^{\left(\Delta_{2}+\Delta_{3}-\Delta_{1}\right) / 2} X_{31}^{\left(\Delta_{3}+\Delta_{1}-\Delta_{2}\right) / 2}},
\end{aligned}
$$

which reproduces the classic results in real space [70], if we use that $X_{a b}=-\frac{1}{2}\left(x_{a}-x_{b}\right)^{2}$. Similarly, the four-point function of identical scalars, of dimensions $\Delta_{a} \equiv \Delta$, is

$$
\left\langle O_{1} O_{2} O_{3} O_{4}\right\rangle=\frac{1}{X_{12}^{\Delta} X_{34}^{\Delta}} g(U, V), \quad \begin{aligned}
& U \\
& \equiv \frac{X_{12} X_{34}}{X_{13} X_{24}}, \\
& V \equiv \frac{X_{14} X_{32}}{X_{13} X_{24}},
\end{aligned}
$$

where $g$ is an arbitrary function of the cross ratios $U$ and $V$.

The real benefit of going to embedding space becomes most manifest for operators with spin. For example, the two-point function of spin- $S$ operators (of dimension $\Delta$ ) takes the form

$$
\left\langle O_{1}^{(S)} O_{2}^{(S)}\right\rangle=\left(Z_{1} \cdot Z_{2}-\frac{Z_{1} \cdot X_{2} Z_{2} \cdot X_{1}}{X_{12}}\right)^{S}\left\langle O_{1} O_{2}\right\rangle
$$

where $\left\langle O_{1} O_{2}\right\rangle$ is given by (3.13) and the relative coefficient in the prefactor is fixed by transversality. Similarly, the three-point function of two scalars (with dimensions $\Delta_{1}$ and $\Delta_{2}$ ) and a spin- $S$ operator (with dimension $\Delta_{3}$ ) is

$$
\left\langle O_{1} O_{2} O_{3}^{(S)}\right\rangle=\left(\frac{\left(Z_{3} \cdot X_{1}\right)\left(X_{2} \cdot X_{3}\right)-\left(Z_{3} \cdot X_{2}\right)\left(X_{1} \cdot X_{3}\right)}{\left(X_{12} X_{13} X_{23}\right)^{1 / 2}}\right)^{S}\left\langle O_{1} O_{2} O_{3}\right\rangle
$$


where $\left\langle\mathrm{O}_{1} \mathrm{O}_{2} \mathrm{O}_{3}\right\rangle$ is given by (3.14). These simple examples already illustrate the power of the embedding space formalism. The above results are simply the most general Lorentzinvariant functions that are consistent with the scaling symmetry (3.11) and the transversality of the operators. More general spinning correlators are obtained in the same way and can be written in terms of a simple basis of tensor structures (see e.g. [63, 68]), although this will not be needed in this paper. For specific weights, the operators become conserved and the correlators satisfy additional constraints [68, 71]. We will study these cases in a separate publication [57].

In [63], conformally-invariant spin- $S_{1}$-spin- $S_{2}$-spin- $S$ three-point functions were written as derivatives of scalar-scalar-spin- $S$ three-point functions:

$$
\left\langle O_{1}^{\left(S_{1}\right)} O_{2}^{\left(S_{2}\right)} O_{3}^{(S)}\right\rangle=\mathcal{D}\left\langle O_{1} O_{2} O_{3}^{(S)}\right\rangle
$$

where the differential operator $\mathcal{D}$ can be written in terms of spin-raising operators acting on $O_{1}$ and $O_{2}$. Relevant differential operators in this construction are

$$
\begin{aligned}
D_{11} & \equiv\left(X_{1} \cdot X_{2}\right) Z_{1} \cdot \frac{\partial}{\partial X_{2}}-\left(Z_{1} \cdot X_{2}\right) X_{1} \cdot \frac{\partial}{\partial X_{2}}-\left(Z_{1} \cdot Z_{2}\right) X_{1} \cdot \frac{\partial}{\partial Z_{2}}+\left(X_{1} \cdot Z_{2}\right) Z_{1} \cdot \frac{\partial}{\partial Z_{2}} \\
D_{12} & \equiv\left(X_{1} \cdot X_{2}\right) Z_{1} \cdot \frac{\partial}{\partial X_{1}}-\left(Z_{1} \cdot X_{2}\right) X_{1} \cdot \frac{\partial}{\partial X_{1}}+\left(Z_{1} \cdot X_{2}\right) Z_{1} \cdot \frac{\partial}{\partial Z_{1}}
\end{aligned}
$$

as well as two more operators with 1 and 2 interchanged. Acting with $D_{a b}$ on a correlator increases the spin at point $a$ by one unit and decreases the dimension at point $b$ by one unit. ${ }^{4}$ In appendix A, these weight-shifting operators, and others, will be discussed in more detail. We will now show how these operators can be utilized to raise the internal spin of the exchanged particles in cosmological correlation functions.

\section{Exchange of spinning particles}

A remarkable feature of the cosmological bootstrap is the fact that all spin-exchange solutions can be obtained from the scalar-exchange solution to (2.13) through the action of a spin-raising operator:
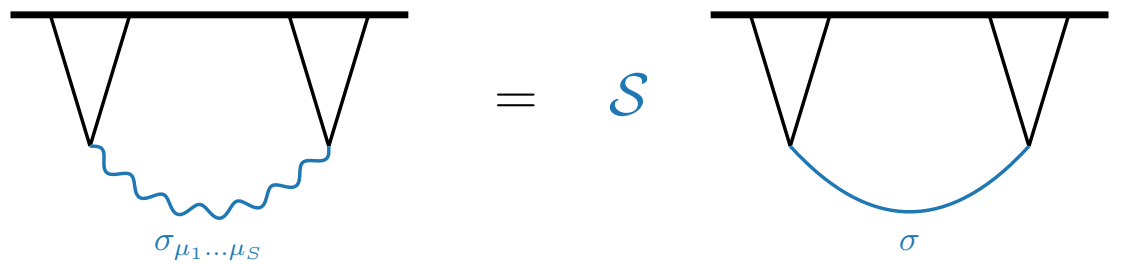

In this section, we will use the embedding space formalism to provide a simple derivation of the relevant spin-raising operator $\mathcal{S}$. This operator is an example of a larger class of weight-shifting operators, which we discuss in more detail in appendix A.

\footnotetext{
${ }^{4}$ This is easy to see by counting factors of $X$ and $Z$. The detailed form of these operators is fixed by demanding that their action preserves the Euclidean section of the projective lightcone.
} 


\subsection{Spin-raising operator}

Ultimately, our goal is to raise the spin of exchanged particles in the scalar four-point functions. To understand the origin of the relevant spin-raising operator, however, it is helpful to first consider raising the spin of an operator in a three-point function. Consider, for concreteness, the correlator of two scalar operators $\varphi$ (with dimension $\Delta_{\varphi}=2$ ) and a generic scalar operator $O$ (with dimension $\Delta_{3}$ ). Using the expression for a general threepoint function (3.14), specialized to this case, we have

$$
\langle\varphi \varphi O\rangle=\left(X_{12}^{4-\Delta_{3}} X_{23}^{\Delta_{3}} X_{31}^{\Delta_{3}}\right)^{-1 / 2} .
$$

Acting on this correlator with the operator defined in (3.20), we find

$$
\left\langle\varphi \tilde{\varphi} O^{(1)}\right\rangle=-\frac{2}{\Delta_{3}} D_{32}\langle\varphi \varphi O\rangle,
$$

where $\tilde{\varphi}$ is the shadow of $\varphi$, which has dimension $\Delta_{\tilde{\varphi}}=d-\Delta_{\varphi}=1$ (for $d=3$ ), and $O^{(1)}$ is a spin-1 operator. The relation in (4.2) is easily confirmed using the results of section 3.3. As advertised, the operator $D_{32}$ raises the spin of the operator at position 3 by one unit and lowers the dimension at position 2 by one unit. It is straightforward to translate the operator $D_{32}$ from embedding space to flat space and then write it in momentum space, where it can be applied to cosmological correlators. Applying these transformations to the operator in (3.20), we find (see appendix B)

$$
D_{32}=z_{3}^{i}\left[\left(\Delta_{3}+S_{3}-1\right) K_{32}^{i}+\frac{1}{2} k_{3}^{i} K_{32}^{j} K_{32}^{j}\right], \quad \text { with } \quad K_{32}^{i} \equiv \partial_{k_{3}^{i}}-\partial_{k_{2}^{i}} .
$$

Finally, we preform a shadow transform to raise the dimension of the operator $\tilde{\varphi}$ at position 2. In Fourier space, this simply amounts to multiplication by $k_{2}$, so that

$$
\left\langle\varphi \varphi O^{(1)}\right\rangle=k_{2}\left\langle\varphi \tilde{\varphi} O^{(1)}\right\rangle=-\frac{2}{\Delta_{3}} k_{2} D_{32}\langle\varphi \varphi O\rangle \equiv-\frac{2}{\Delta_{3}} i \mathcal{S}_{12}\langle\varphi \varphi O\rangle,
$$

where we have defined the spin-raising operator $\mathcal{S}_{12}$ that implements the combined action of (4.3) and the shadow transform. For our future uses, it is convenient to use momentum conservation to write the differential operator (4.3) in terms of $\vec{k}_{1}$ and $\vec{k}_{2}$, rather than $\vec{k}_{3}$. The differential operator then acts on the fields at positions 1 and 2, which explains the subscript of $\mathcal{S}_{12}$. Repeated application of $i \mathcal{S}_{12}=k_{2} D_{32}$ would raise the spin further.

\subsection{Raising internal spin}

We can use the operator $\mathcal{S}_{12}$ to raise the spin of the exchanged field using the scalarexchange solution as a seed. We first show how this works in detail for spins 1 and 2 and then discuss the generalization to arbitrary spin.

\subsubsection{Spin-1 exchange}

We first consider the mapping of the scalar-exchange solution to a spin-1 exchange solution. To understand which spin-raising operator to use, it is helpful to first consider 
the disconnected contribution to the four-point function coming from spin-1 exchange (see e.g. appendix A of [1]):

$$
\langle\varphi \varphi \varphi \varphi\rangle_{\mathrm{d}}^{(1)}=\frac{\left\langle\varphi_{\vec{k}_{1}} \varphi_{\vec{k}_{2}} O_{-\vec{s}}^{i}\right\rangle\left(\Pi_{1}\right)_{i j}\left\langle O_{\vec{s}}^{j} \varphi_{\vec{k}_{3}} \varphi_{\vec{k}_{4}}\right\rangle}{\left\langle O_{\vec{s}} O_{-\vec{s}}\right\rangle},
$$

where the symmetric traceless tensor $\left(\Pi_{1}\right)_{i j}$ contains the polarization structure of the inverse two-point function of the exchanged field: ${ }^{5}$

$$
\left\langle O_{\vec{s}}^{i} O_{-\vec{s}}^{j}\right\rangle^{-1} \propto\left(\Pi_{1}\right)^{i j}\left\langle O_{\vec{s}} O_{-\vec{s}}\right\rangle^{-1} .
$$

Using the spin-raising operator in (4.4), the expression (4.5) can be written as

$$
\langle\varphi \varphi \varphi \varphi\rangle_{\mathrm{d}}^{(1)}=-\left(\Pi_{1}\right)_{i j} \frac{\mathcal{S}_{12}^{i}\left\langle\varphi_{\vec{k}_{1}} \varphi_{\vec{k}_{2}} O_{-\vec{s}}\right\rangle \mathcal{S}_{34}^{j}\left\langle O_{\vec{s}} \varphi_{\vec{k}_{3}} \varphi_{\vec{k}_{4}}\right\rangle}{\left\langle O_{\vec{s}} O_{-\vec{s}}\right\rangle},
$$

where $O$ is a scalar operator of dimension $\Delta$. From this, it is clear that the operator $\left(\Pi_{1}\right)_{i j} \mathcal{S}_{12}^{i} \mathcal{S}_{34}^{j}$ acting in the numerator raises the spin of the exchanged particle. Our goal is to simplify this operator such that we can pull it outside and have it act on the total disconnected correlator $\langle\varphi \varphi \varphi \varphi\rangle_{\mathrm{d}}^{(0)}$.

We start by simplifying the expression $\mathcal{S}_{12}\langle\varphi \varphi O\rangle$. Since the three-point function depends only on the magnitudes of the momenta, we can write the operator in (4.4) as

$$
\mathcal{S}_{12}^{i}=(\Delta-1) k_{2}^{i} \frac{\partial}{\partial k_{2}}+k_{2} s^{i}\left[(\Delta-2) \frac{1}{s} \frac{\partial}{\partial s}-\frac{1}{k_{2}} \frac{\partial}{\partial k_{2}}-\frac{1}{2} \frac{\partial^{2}}{\partial k_{2}^{2}}-\frac{1}{2} \frac{\partial^{2}}{\partial s^{2}}-\left(\hat{k}_{2} \cdot \hat{s}\right) \frac{\partial^{2}}{\partial k_{2} \partial s}\right],
$$

where we have used $\vec{s}=\vec{k}_{1}+\vec{k}_{2}$, which is the momentum of the exchanged particle. Next, we change variables from $k_{1}, k_{2}$ to

$$
u=\frac{s}{k_{1}+k_{2}}, \quad \hat{\alpha}=\frac{k_{1}-k_{2}}{s} .
$$

In these variables, the scalar three-point function has an extremely simple functional dependence [1]: $\langle\varphi \varphi O\rangle=s^{\Delta-2} \hat{f}(u)$, so we can drop any derivatives with respect to $\hat{\alpha}$ and derivatives with respect to $s$ act simply on the polynomial prefactor. In simplifying the answer it is convenient to use $\Delta_{u} \hat{f}(u)=(\Delta-1)(\Delta-2) \hat{f}(u)$, where $\Delta_{u}$ was defined below (2.11). After some algebra, the operator (4.8) simplifies to

$$
\mathcal{S}_{12}^{i}=\frac{\Delta-1}{2 s}\left[\alpha^{i} u^{2} \partial_{u}-s^{i} \hat{\alpha}\left(u \partial_{u}+\Delta-2\right)\right],
$$

where we have introduced $\vec{\alpha} \equiv \vec{k}_{1}-\vec{k}_{2}$, for later convenience. ${ }^{6}$ Importantly, this operator only involves derivatives with respect to $u$. Similar manipulations let us simplify the operator $\mathcal{S}_{34}$. All we have to do is replace $\vec{s}$ by $-\vec{s}, \vec{\alpha}$ by $\vec{\beta}=\vec{k}_{3}-\vec{k}_{4}$ and $u, \hat{\alpha}$ by $v, \hat{\beta}$. The resulting operator will only contain derivatives with respect to $v$.

\footnotetext{
${ }^{5}$ Note that in Fourier space, the inverse of the two-point function is equivalent to the two-point function of an operator with the shadow dimension (suitably normalized).

${ }^{6}$ The magnitude of this vector is not to be confused with $\hat{\alpha}=\left(k_{1}-k_{2}\right) / s$, i.e. $|\vec{\alpha}| / s \neq \hat{\alpha}$.
} 
Having simplified the differential operators, we now turn our attention to the polarization structure of the exchanged operator. For exchange of an operator of weight $\Delta$, the spin-1 polarization tensor is (see appendix $\mathrm{C}$ for a derivation)

$$
\left(\Pi_{1}\right)_{i j}=\pi_{i j}+\frac{1-\Delta}{\Delta-2} \hat{s}_{i} \hat{s}_{j}
$$

where $\pi_{i j} \equiv \delta_{i j}-\hat{s}_{i} \hat{s}_{j}$ is a transverse projector. Substituting (4.10) and (4.11) into (4.7), we see that we can pull the differential operators outside to obtain

$$
\langle\varphi \varphi \varphi \varphi\rangle_{\mathrm{d}}^{(1)} \propto\left(\Pi_{1,1} D_{u v}+\Pi_{1,0} \Delta_{u}\right)\langle\varphi \varphi \varphi \varphi\rangle_{\mathrm{d}}^{(0)},
$$

where we have defined the differential operator $D_{u v} \equiv(u v)^{2} \partial_{u} \partial_{v}$ and the polarization sums $\Pi_{1,1} \equiv \alpha^{i} \pi_{i j} \beta^{j} / s^{2}$ and $\Pi_{1,0} \equiv \hat{\alpha} \hat{\beta}$. The differential operator that raises the spin of the exchanged particle from zero to one therefore is

$$
\mathcal{S}_{u v}^{(1)} \equiv \Pi_{1,1} D_{u v}+\Pi_{1,0} \Delta_{u}
$$

which is precisely the result found in [1].

Thus far, we have only discussed the disconnected contribution to the four-point function arising from the exchange of a single scalar operator of general conformal weight $\Delta$. To relate this to the most general exchange four-point function, we note that any fourpoint function can be decomposed as a sum over the exchange of various states of different conformal weights, but fixed spin. Moreover, since the spin-raising operator $\mathcal{S}_{u v}^{(1)}$ in $(4.13)$ does not depend on conformal dimension, acting with it on the complete scalar exchange solution $\hat{F}^{(0)}$ produces the complete spin-1 exchange solution $\hat{F}^{(1)}$ :

$$
\hat{F}^{(1)}=\mathcal{S}_{u v}^{(1)} \hat{F}^{(0)} .
$$

Indeed, this relation is precisely what was found by other means in [1].

\subsubsection{Spin-2 exchange}

We can repeat the same exercise for spin-2 exchange, which is algebraically more involved, but conceptually the same. In this case, the disconnected part of the four-point function is

$$
\langle\varphi \varphi \varphi \varphi\rangle_{\mathrm{d}}^{(2)}=\frac{\left\langle\varphi_{\vec{k}_{1}} \varphi_{\vec{k}_{2}} O_{-\vec{s}}^{i j}\right\rangle\left(\Pi_{2}\right)_{i j, l m}(\hat{s})\left\langle O_{\vec{s}}^{l m} \varphi_{\vec{k}_{3}} \varphi_{\vec{k}_{4}}\right\rangle}{\left\langle O_{\vec{s}} O_{-\vec{s}}\right\rangle},
$$

where $\left(\Pi_{2}\right)_{i j, l m}$ is the spin-2 polarization tensor. Like in the spin-1 case, we can write this in terms of the spin-raising operator (4.4) acting on scalar three-point functions

$$
\langle\varphi \varphi \varphi \varphi\rangle_{\mathrm{d}}^{(2)}=\left(\Pi_{2}\right)_{i j, l m} \frac{\left(\mathcal{S}_{12}^{2}\right)^{i j}\left\langle\varphi_{\vec{k}_{1}} \varphi_{\vec{k}_{2}} O_{-\vec{s}}\right\rangle\left(\mathcal{S}_{34}^{2}\right)^{l m}\left\langle O_{\vec{s}} \varphi_{\vec{k}_{3}} \varphi_{\vec{k}_{4}}\right\rangle}{\left\langle O_{\vec{s}} O_{-\vec{s}}\right\rangle} .
$$

As before, we want to write the spin-raising operators in such way that we can act with them on the entire disconnected correlator. This proceeds very similarly to the spin- 1 case. 
We first focus on $\mathcal{S}_{12}^{2}\langle\varphi \varphi O\rangle$. Changing variables to $u, \hat{\alpha}, \vec{\alpha}$ and eliminating $s$-derivatives, we can write the spin-raising operator as

$$
\begin{aligned}
\left(\mathcal{S}_{12}^{2}\right)^{i j} \propto & \frac{\Delta(\Delta-1)}{s^{2}}\left[\delta^{i j} s^{2}\left[3\left(1-\hat{\alpha}^{2}\right) u \partial_{u}+(\Delta-2)\left(\Delta-3 \hat{\alpha}^{2}\right)\right]\right. \\
& -3 \alpha^{i} \alpha^{j} u^{2} \partial_{u}\left(u^{2} \partial_{u}\right)+3\left(\alpha^{i} s^{j}+s^{i} \alpha^{j}\right) u \hat{\alpha}\left[\Delta-3+\partial_{u}\left(u^{2} \partial_{u}\right)\right] \\
& \left.-3 s^{i} s^{j}\left(\Delta-2+u \partial_{u}+\hat{\alpha}^{2}(\Delta-2)(\Delta-4)+\hat{\alpha}^{2}(2 \Delta-5) u \partial_{u}+\hat{\alpha}^{2} u^{2} \partial_{u}^{2}\right)\right],
\end{aligned}
$$

where again all derivatives are only with respect to $u$. As in the spin-1 case, we can get $\mathcal{S}_{34}^{2}$ from the replacement $\{\vec{s}, u, \hat{\alpha}, \vec{\alpha}\} \mapsto\{-\vec{s}, v, \hat{\beta}, \vec{\beta}\}$. Importantly, these differential operators again have no $s$-derivatives, so we can pull them outside to act on the full disconnected correlator $\langle\varphi \varphi \varphi \varphi\rangle_{\mathrm{d}}^{(0)}$.

Next, we consider the spin-2 polarization structure, which is given by (see appendix C)

$$
\left(\Pi_{2}\right)_{i j, l m}=\left(\Pi_{2,2}\right)_{i j, l m}-\frac{\Delta}{\Delta-3}\left(\Pi_{2,1}\right)_{i j, l m}+\frac{\Delta(\Delta-1)}{(\Delta-2)(\Delta-3)}\left(\Pi_{2,0}\right)_{i j, l m}
$$

where we have defined the individual helicity components as

$$
\begin{aligned}
& \left(\Pi_{2,2}\right)^{i j}{ }_{l m}=\pi_{(l}^{(i} \pi_{m)}^{j j}-\frac{1}{2} \pi^{i j} \pi_{l m}, \\
& \left(\Pi_{2,1}\right)^{i j}{ }_{l m}=2 \hat{s}^{(i} \hat{s}_{(l} \pi_{m)}^{j)}, \\
& \left(\Pi_{2,0}\right)^{i j}{ }_{l m}=\frac{3}{2}\left(\hat{s}^{i} \hat{s}^{j}-\frac{1}{3} \delta^{i j}\right)\left(\hat{s}_{l} \hat{s}_{m}-\frac{1}{3} \delta_{l m}\right) .
\end{aligned}
$$

Combining these expressions together with the simplified expressions for $\mathcal{S}_{12}^{2}$ and $\mathcal{S}_{34}^{2}$, and performing some algebra, then gives

$$
\langle\varphi \varphi \varphi \varphi\rangle_{\mathrm{d}}^{(2)} \propto\left(\Pi_{2,2} D_{u v}^{2}+\Pi_{2,1} D_{u v}\left(\Delta_{u}-2\right)+\Pi_{2,0} \Delta_{u}\left(\Delta_{u}-2\right)\right)\langle\varphi \varphi \varphi \varphi\rangle_{\mathrm{d}}^{(0)},
$$

where we have defined the following polarization sums

$$
\begin{aligned}
\Pi_{2,2} & \equiv \frac{3}{2 s^{4}} \alpha_{i} \alpha_{j}\left(\Pi_{2,2}\right)^{i j}{ }_{l m} \beta^{l} \beta^{m}, \\
\Pi_{2,1} & \equiv \frac{3}{s^{2}} \hat{\alpha} \hat{\beta} \alpha^{i} \pi_{i j} \beta^{j}, \\
\Pi_{2,0} & \equiv \frac{1}{4}\left(1-3 \hat{\alpha}^{2}\right)\left(1-3 \hat{\beta}^{2}\right) .
\end{aligned}
$$

We have therefore found an operator that raises the spin of the exchanged particle from zero to two:

$$
\mathcal{S}_{u v}^{(2)} \equiv \Pi_{2,2} D_{u v}^{2}+\Pi_{2,1} D_{u v}\left(\Delta_{u}-2\right)+\Pi_{2,0} \Delta_{u}\left(\Delta_{u}-2\right) .
$$

For the same reason as before, we can take this operator and apply it to the full connected four-point function to obtain the massive spin-2 exchange solution

$$
\hat{F}^{(2)}=\mathcal{S}_{u v}^{(2)} \hat{F}^{(0)},
$$

which is the same result as in [1]. 


\subsubsection{Higher-spin exchange}

The spin-raising procedure we have described is completely algorithmic and can therefore easily be extended to arbitrary spin. In particular, the spin- $S$ exchange solution can be written schematically as

$$
\hat{F}^{(S)}=\left(\Pi_{S}\right)_{i_{1} \ldots i_{S}, j_{1} \ldots j_{S}}\left(\mathcal{S}_{12}^{S}\right)^{i_{1} \ldots i_{S}}\left(\mathcal{S}_{34}^{S}\right)^{j_{1} \ldots j_{S}} \hat{F}^{(0)},
$$

where $\mathcal{S}_{a b}^{S}$ are the spin- $S$ analogues of $(4.10)$ and $(4.17),\left(\Pi_{S}\right)_{i_{1} \ldots, j_{1} \ldots}$ is the polarization structure of the spin- $S$ two-point function, and $\hat{F}^{(0)}$ is the same seed function as before.

It is instructive to write the spin-raising operator as a sum over the different helicity components

$$
\hat{F}^{(S)}=\sum_{m=0}^{S} \Pi_{S, m} \mathcal{D}_{u v}^{(S, m)} \hat{F}^{(0)}
$$

where the differential operators at each helicity $m$ are given by

$$
\mathcal{D}_{u v}^{(S, m)}=D_{u v}^{m} \prod_{j=1}^{S-m}\left(\Delta_{u}-(S-j)(S-j+1)\right) .
$$

The polarization sums appearing in (4.25) are presented in detail in appendix C. Specializing to $d=3$ dimensions, they can be written as

$$
\Pi_{S, m}=\left(2-\delta_{m 0}\right)(-\hat{L})^{m} \cos (m \psi) \tilde{P}_{S}^{m}(\hat{\alpha}) \tilde{P}_{S}^{-m}(\hat{\beta}),
$$

where $\tilde{P}_{S}^{m}(x) \equiv\left(1-x^{2}\right)^{-|m| / 2} P_{S}^{m}(x)$ is a modified version of the associated Legendre polynomial $P_{S}^{m}(x)$, and $\psi$ is the angle between $\hat{k}_{1}$ and $\hat{k}_{3}$ on the plane perpendicular to $\hat{s}$, given by

$$
\cos \psi=\frac{\cos \gamma-\cos \theta_{1} \cos \theta_{3}}{\sin \theta_{1} \sin \theta_{3}}=\frac{\hat{T}}{\hat{L}}
$$

In this expression, we have defined the angles $\cos \gamma \equiv \hat{k}_{1} \cdot \hat{k}_{3}, \cos \theta_{a} \equiv \hat{k}_{a} \cdot \hat{s}$, and the kinematic invariants:

$$
\begin{aligned}
\hat{T} & \equiv \frac{\alpha_{i} \pi^{i j} \beta_{j}}{s^{2}}=\hat{\tau}+\frac{\hat{\alpha} \hat{\beta}}{u v}, \\
\hat{L}^{2} & \equiv \frac{\alpha_{i} \pi^{i j} \alpha_{j} \beta_{k} \pi^{k l} \beta_{l}}{s^{4}}=\frac{\left(1-u^{2}\right)\left(1-v^{2}\right)}{u^{2} v^{2}}\left(1-\hat{\alpha}^{2}\right)\left(1-\hat{\beta}^{2}\right) .
\end{aligned}
$$

The overall normalization was chosen such that $\Pi_{0,0}=1$.

Note that some of the "angles" appearing in (4.27) are slightly unusual. However, taking the collapsed limit, $s \rightarrow 0$, the angular dependence simply becomes

$$
\lim _{s \rightarrow 0} \Pi_{S, m}=\left(2-\delta_{m 0}\right)(-u v)^{m} \cos (m \psi) P_{S}^{m}\left(\cos \theta_{1}\right) P_{S}^{-m}\left(\cos \theta_{3}\right) .
$$

Along with the momentum dependence (2.16), this angular dependence in the collapsed limit is one of the hallmarks of the exchange of massive particles with spin. The characteristic angular structure allows both mass and spin spectroscopy to be performed using the collapsed limit of the four-point function (or the squeezed limit of the three-point function). 


\section{$5 \quad$ Inflationary three-point functions}

So far, we have presented results for the four-point functions of $\Delta=2$ operators in de Sitter space. To describe inflationary correlators, however, we further need to raise the weight of the external operators to $\Delta=3$, corresponding to a massless field in the bulk. This field plays the role of the inflaton in conventional models of slow-roll inflation.

In [1], a set of weight-raising operators was introduced, relating the four-point functions of conformally-coupled and massless scalars. The derivation of these operators, however, was somewhat unsatisfactory because they had to be found separately for each spinexchange solution $\hat{F}^{(S)}$. Explicit results were therefore only presented for low spins. In this section, we will show that the embedding space formalism allows for very simple derivation of a single weight-raising operator:

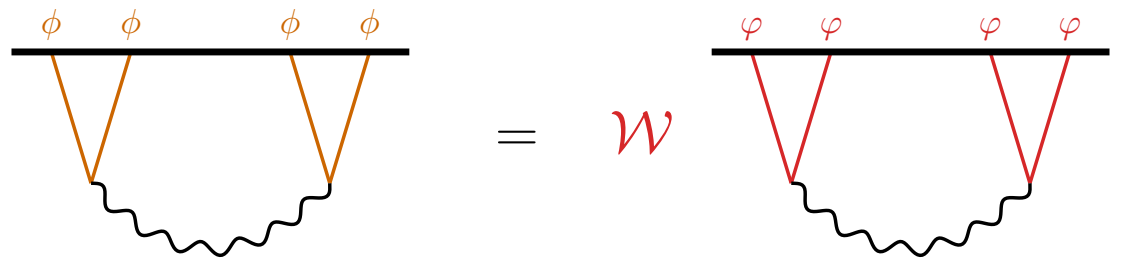

Acting with this operator $\mathcal{W}$ on the solutions $\hat{F}^{(S)}$ straightforwardly reproduces the results in [1] for low spin, and automatically generalizes them to arbitrary spin.

Finally, we will show how to perturb our de Sitter four-point functions to obtain inflationary three-point functions. We will present explicit formulas for the source functions appearing in these inflationary correlators for any mass and spin of the exchanged field.

\subsection{Weight-raising operator}

Recall from (3.15) that, in embedding space, the most general four-point functions of a $\Delta=2$ scalar operator, $\varphi$, and a $\Delta=3$ scalar operator, $\phi$, can be expressed as ${ }^{7}$

$$
\begin{aligned}
\langle\varphi \varphi \varphi \varphi\rangle & =\frac{1}{X_{12}^{2} X_{34}^{2}} f(U, V), \\
\langle\phi \phi \phi \phi\rangle & =\frac{1}{X_{12}^{3} X_{34}^{3}} h(U, V),
\end{aligned}
$$

where $f$ and $h$ are arbitrary functions of the cross ratios $U$ and $V$. It is then straightforward to show that the following relation holds

$$
\langle\phi \phi \phi \phi\rangle=\mathcal{W}_{12} \mathcal{W}_{34}\langle\varphi \varphi \varphi \varphi\rangle,
$$

where we have defined

$$
\mathcal{W}_{a b} \equiv \eta^{M N}\left(\frac{\partial}{\partial X_{a}^{M}}+\frac{X_{a, M}}{3} \frac{\partial^{2}}{\partial X_{a}^{2}}\right)\left(\frac{\partial}{\partial X_{b}^{N}}+\frac{X_{b, N}}{3} \frac{\partial^{2}}{\partial X_{b}^{2}}\right) .
$$

We see that the operator $\mathcal{W}_{12} \mathcal{W}_{34}$ acts as a weight-raising operator. The structure of the operators in (5.4) acting on the points $a$ and $b$ can be understood as follows: to lower the

\footnotetext{
${ }^{7}$ Recently, the momentum space versions of these formulas have been presented in [33].
} 
conformal weight by one unit, one must reduce the overall power of $X$ by one unit. This is done by taking derivatives with respect to $X$. On the projective lightcone, there are only two possible operators that reduce the weight by one, namely $\partial / \partial X$ and $X \partial^{2} / \partial X^{2}$. The specific linear combination of these two operators in (5.4) is the unique combination that preserves the Euclidean section. More details of the construction of these weight-shifting operators can be found in appendix A.

\subsection{Raising external weight}

Our task now is simply to project (5.4) to position space and then transform the result to momentum space, so that we can act on our solutions for $\langle\varphi \varphi \varphi \varphi\rangle$ to produce the corresponding correlators $\langle\phi \phi \phi \phi\rangle$. For $\Delta_{1}=\Delta_{2}=2$, we find (see appendix B)

$$
\mathcal{W}_{12}=\frac{\left(k_{1} k_{2}\right)^{2}}{2} \vec{K}_{12} \cdot \vec{K}_{12}-\vec{k}_{1} \cdot \vec{k}_{2}-\left(k_{2}^{2} \vec{k}_{1} \cdot \vec{K}_{12}+1 \leftrightarrow 2\right),
$$

where $\vec{K}_{12}$ was defined as in (4.3). To apply the above weight-shifting operator to the spinexchange four-point function (4.25), it is helpful to express it in terms of the dimensionless kinematic variables $\{s, u, v, \hat{\alpha}, \hat{\beta}, \hat{\tau}\}$. Although this is conceptually straightforward, it is algebraically somewhat involved. After some work, we can express (5.5) as

$$
\begin{aligned}
\mathcal{W}_{12}= & \frac{s^{2}}{8 u^{2}}\left[2 \left(1-u^{2}\left(2-\hat{\alpha}^{2}\right)+2 u\left(2-3 u^{2}+u^{4} \hat{\alpha}^{2}\right) \partial_{u}+u^{2}\left(1-u^{2}\right)\left(1-u^{2} \hat{\alpha}^{2}\right) \partial_{u}^{2}\right.\right. \\
& -2 \hat{\alpha}\left(1-u^{2}\left(2-\hat{\alpha}^{2}\right)\right) \partial_{\hat{\alpha}}+\left(1-\hat{\alpha}^{2}\right)\left(1-u^{2} \hat{\alpha}^{2}\right) \partial_{\hat{\alpha}}^{2}+\frac{v\left(1-\hat{\alpha}^{2} u^{2}\right)^{2}\left(1-v^{2}\left(1-\hat{\beta}^{2}\right)\right)}{u^{2} v^{2}} \partial_{\hat{\tau}}^{2} \\
& \left.-\frac{4 u(\hat{\alpha} u(2 \hat{\beta}+\hat{\alpha} \hat{\tau} u v)+v \hat{\tau})+2\left(1-\hat{\alpha}^{2} u^{2}\right)\left(u^{2}(\hat{\alpha} \hat{\beta} u+v \hat{\tau}) \partial_{u}+(\hat{\beta}+\hat{\alpha} \hat{\tau} u v) \partial_{\hat{\alpha}}\right)}{u v} \partial_{\hat{\tau}}\right],
\end{aligned}
$$

where $\hat{\alpha}, \hat{\beta}$ and $\hat{\tau}$ were defined in (2.17).

When acting on spinning correlators in momentum space, it will be useful to decompose $\mathcal{W}_{12}$ into helicity components. We do this by commuting it through the polarization structure as

$$
\mathcal{W}_{12} \sum_{m=0}^{S} \Pi_{S, m} \mathcal{D}_{u v}^{(S, m)} \hat{F}^{(0)}=\frac{s^{2}}{2} \sum_{m=0}^{S} \Pi_{S, m} U_{12}^{(S, m)} \mathcal{D}_{u v}^{(S, m)} \hat{F}^{(0)},
$$

where $U_{12}^{(S, m)}$ is a dimensionless, helicity-decomposed weight-shifting operator. Using the detailed form of the polarization structure (4.27), we find that this operator is given by

$$
\begin{aligned}
U_{12}^{(S, m)} \equiv & \left(1+\frac{u\left(1-\hat{\alpha}^{2} u^{2}\right)}{4} \partial_{u}\right) \frac{\left(1-u^{2}\right)\left(1+u \partial_{u}\right)-2 S}{u^{2}}+(S+m) \hat{\alpha} \frac{P_{S-1}^{m}(\hat{\alpha})}{P_{S}^{m}(\hat{\alpha})} \\
& +\frac{(S-m)\left[(S+m+1) u^{2} \hat{\alpha}^{2}-S-m+3+2 u\left(1-u^{2} \hat{\alpha}^{2}\right) \partial_{u}\right]}{4 u^{2}}
\end{aligned}
$$

where $P_{-1}^{0}(\hat{\alpha})=1$. This agrees with the results of [1] for $S=1,2$, but now holds for all $S$. The corresponding operator $U_{34}^{(S, m)}$ is obtained from this expression by swapping the kinematic variables $\{u, \hat{\alpha}\} \mapsto\{v, \hat{\beta}\}$. 
Using the operators $U_{12}^{(S, m)}$ and $U_{34}^{(S, m)}$, the general spin-exchange four-point function of massless scalars can then be written in the following form

$$
F_{\Delta=3}^{(S)}=s^{3} \sum_{m=0}^{S} \Pi_{S, m} U_{12}^{(S, m)} U_{34}^{(S, m)} \mathcal{D}_{u v}^{(S, m)} \hat{F}_{\Delta=2}^{(0)} .
$$

As we will show in the next section, only the longitudinal $(m=0)$ component contributes to inflationary three-point functions. In that case, the operator in (5.8) can be written in a slightly simplified form as

$$
U_{12}^{(S, 0)}=\frac{\left(1-u^{2} \hat{\alpha}^{2}\right)\left(\Delta_{u}-(S-1)(S+2)\right)}{4 u^{2}}+\frac{1-u^{2}}{u} \partial_{u}+S \hat{\alpha} \frac{P_{S-1}(\hat{\alpha})}{P_{S}(\hat{\alpha})}-1+(1-S) \hat{\alpha}^{2} .
$$

This operator acts on the longitudinal part of the $\Delta=2$ solution,

$$
\hat{F}_{L}^{(S)} \equiv \mathcal{D}_{u v}^{(S, 0)} \hat{F}^{(0)}=\prod_{j=1}^{S}\left(\Delta_{u}-(S-j)(S-j+1)\right) \hat{F}^{(0)},
$$

where the operator $\mathcal{D}_{u v}^{(S, 0)}$ was defined in (4.26).

\subsection{From de Sitter to inflation}

Using the operator $U_{12}^{(S, m)}$ defined in (5.8), we are able to efficiently generate $\Delta=3$ scalar solutions to the conformal Ward identities. To apply these results to inflation, we must take into account that the inflaton field is not exactly massless and has a time-dependent expectation value $\bar{\phi}(t)$. We assume that the associated breaking of the de Sitter symmetries is weak and can be treated perturbatively. As explained in [17, 73], inflationary three-point functions to leading order in slow-roll can then be obtained from our de Sitter four-point functions by the following procedure:

- First, perturb the scaling dimensions of the external fields, $\Delta=3 \mapsto 3-\epsilon$, where $\epsilon \ll 1$ is the slow-roll parameter.

- Second, take the soft limit of one of the external momenta (which we take to be $k_{4} \rightarrow 0$ ), and expand the result in powers of $\epsilon$.

From the bulk point of view, this amounts to evaluating one of the external fields $\phi$ on its time-dependent background value $\bar{\phi}(t)$. For shift-symmetric inflaton interactions, ${ }^{8}$ this gives a three-point vertex with a coupling proportional to $\dot{\bar{\phi}}$. In slow-roll inflation, this coupling is almost constant and can be related to the slow-roll parameter $\epsilon$. This allows us to identify the soft limit of the perturbed de Sitter four-point function with the corresponding three-point function in slow-roll inflation.

\footnotetext{
${ }^{8}$ For non-derivatively coupled interactions, like $\phi^{4}$, the expectation values will generically contain logarithms and not be de Sitter invariant. However, the breaking of de Sitter symmetry is mild and the violation of de Sitter symmetry is given by local terms. These cases have been analyzed carefully in $[1,8,24,34]$.
} 
Since the weight-shifting operator changes the weight by precisely one unit, it makes sense to perturb the conformal dimensions of the seed to $\Delta=2-\epsilon$. To apply the weightraising operator to the perturbed seed correlator, we need the generalization of (5.4) to general $\Delta$ :

$$
\mathcal{W}_{a b} \equiv \eta^{M N}\left(\frac{\partial}{\partial X_{a}^{M}}+\frac{X_{a, M}}{2 \Delta-1} \frac{\partial^{2}}{\partial X_{a}^{2}}\right)\left(\frac{\partial}{\partial X_{b}^{N}}+\frac{X_{b, N}}{2 \Delta-1} \frac{\partial^{2}}{\partial X_{b}^{2}}\right) .
$$

Note that, due to the transversality of the polarization tensors, all of the polarization sums (4.27) except the longitudinal component, $m=0$, vanish in the soft limit. This is easily seen from the $\hat{L}$ dependence of (4.27), which vanishes as $u, \hat{\alpha} \rightarrow 1$ (or $v, \hat{\beta} \rightarrow 1$ ). Repeating the derivation in section 5.2 for $\Delta=2-\epsilon$, and expanding for small $\epsilon$, we obtain the following weight-shifting operator for the longitudinal mode of the four-point function

$$
U_{34}^{(S, 0)} \rightarrow \bar{U}_{34}^{(S, 0)}-\epsilon\left[\frac{3-2(S-1) v \hat{\beta}-\left(4+(2 S-3) \hat{\beta}^{2}\right) v^{2}}{2 v^{2}}+\frac{S(1+v \hat{\beta})}{2 v} \frac{P_{S-1}(\hat{\beta})}{P_{S}(\hat{\beta})}\right],
$$

where $\bar{U}_{34}^{(S, 0)}$ is given by (5.10), and we have only kept the correction at linear order in $\epsilon$.

In the soft limit $k_{4} \rightarrow 0$ (or $v, \hat{\beta} \rightarrow 1$ ), the unperturbed weight-raising operator $\bar{U}_{34}^{(S, 0)}$ vanishes identically (i.e. independent of the correlator it acts on) and we simply get

$$
U_{34}^{(S, 0)} \stackrel{k_{4} \rightarrow 0}{\longrightarrow}-\epsilon
$$

independent of spin. This means that, at order $\epsilon$, we can simply take the seed function to be that of the unperturbed dimension $\Delta=2$ operators, $\hat{F}_{L}^{(S)}(u, 1)$, evaluated at $v=1$, and multiply it by $-\epsilon$. The corresponding inflationary bispectrum is then obtained by applying the operator $U_{12}^{(S, 0)}$ and summing over permutations (to account for the fact that the fourpoint function was evaluated for the $s$-channel exchange). Putting everything together, the inflationary bispectrum for spin- $S$ exchange can then be written as ${ }^{9}$

$$
B^{(S)}\left(k_{1}, k_{2}, k_{3}\right)=-\epsilon k_{3}^{3} P_{S}(\hat{\alpha}) U_{12}^{(S, 0)} \hat{F}_{L}^{(S)}(u, 1)+\text { perms. }
$$

where $U_{12}^{(S, 0)}$ and $\hat{F}_{L}^{(S)}$ were defined in (5.10) and (5.11), respectively, and $P_{S}$ is a Legendre polynomial that arises from the polarization sum $\Pi_{S, 0}$. This formula holds for any $S$, generalizing the result of [1] to all spins.

\subsection{Partially massless exchange}

As a new application of the weight-shifting technology developed in this paper, we construct the inflationary bispectra arising from the exchange of partially massless (PM) fields. These

\footnotetext{
${ }^{9}$ The bispectrum function, $B^{(S)}$, is the correlator of the sub-leading fall-off dual to $\zeta$ and is hence related to the observed bispectrum by

$$
\left\langle\zeta_{\vec{k}_{1}} \zeta_{\vec{k}_{2}} \zeta_{\vec{k}_{3}}\right\rangle=\prod_{a=1}^{3}\left(\frac{H^{2}}{2 M_{\mathrm{pl}}^{2} \epsilon k_{a}^{3}}\right) B^{(S)}\left(k_{1}, k_{2}, k_{3}\right)
$$

where $H$ is the Hubble scale during inflation and $M_{\mathrm{pl}}$ is the (reduced) Planck mass. The prefactor arises from the shadow transform.
} 
PM fields are unitary spinning representations that exist on (anti) de Sitter space, but have no flat-space counterparts [50, 74-79]. They occur at special values of the mass-to-Hubble ratio,

$$
\frac{m^{2}}{H^{2}}=S(S-1)-T(T+1),
$$

where $S$ is the spin and $T \in\{0, \cdots, S-1\}$ is the "depth" of the field. The corresponding dual operators have integer dimensions $\Delta_{\sigma}=2+T$. At these special points, the theory possesses an additional gauge invariance that projects out modes with helicity $\leq T$. The tight structure of PM representations is reflected in the simplicity of their mode functions, which leads to simple analytic expressions for the associated correlation functions.

We are interested in the inflationary three-point functions arising from the exchange of PM fields. As we have seen above, these bispectra only depend on the longitudinal modes of the exchanged particle, which for a PM field isn't a propagating degree of freedom. This does not mean, however, that the corresponding bispectra are trivial. Higher-spin particles have a nontrivial constraint structure, where the non-propagating degrees of freedom are fixed in terms of the propagating ones. In particular, the longitudinal modes mediate Coulomb-like potentials, which lead to distinct imprints in the inflationary bispectra. In order to probe all degrees of freedom of the PM field, we would have to measure inflationary trispectra. Nonetheless, as we will see below, the Coulomb potentials generate bispectrum shapes with striking features that uniquely characterize the presence of a PM field in the spectrum.

The general features of PM exchange are substantially simpler to describe than those of massive exchange. Recall that the longitudinal part of the $\Delta=2$ correlator coming from spin- $S$ exchange is given by (5.11). We can write this in a slightly more illuminating form by redefining $T \equiv S-j$ to obtain

$$
\hat{F}_{L}^{(S)}=\prod_{T=0}^{S-1}\left[\Delta_{u}-T(T+1)\right] \hat{F}_{\Delta_{\sigma}}^{(0)},
$$

where we have added the weight dependence of the seed as a subscript to avoid confusion. Notice that the differential operators appearing on the right-hand side are precisely those appearing in the differential equation describing the exchange of a PM particle of depth $T$ :

$$
\left[\Delta_{u}-T(T+1)\right] \hat{F}_{\Delta_{\sigma}=2+T}^{(0)}=\hat{C}_{0}
$$

It is important to emphasize that a given PM seed $\hat{F}_{\Delta_{\sigma}}^{(0)}$ will only have the right conformal weight to satisfy (5.19) for a particular depth. Since (5.18) includes the left-hand-side of (5.19) for all depths though, this means that at every PM point, one of the operators in (5.18) will reduce the seed exchange function to a contact solution through (5.19). This contact solution is then acted on by the operators in (5.16). It is for this reason that the PM-induced bispectra are so simple.

Cleanly disentangling the effects of PM exchange is somewhat subtle. In the case of massive particle exchange, there are characteristic non-analytic features that cannot be mimicked by contact interactions and therefore are unambiguously attributed to the 
exchange. In the present case, however, the resulting bispectra will be rational functions of momenta, and so it is less obvious which parts to ascribe to particle exchange. In practice, this subtlety can be managed by explicitly subtracting off all possible contact solutions. After performing this subtraction, the leftover shape is a sharp signature of the exchanged PM particle.

Spin-2 exchange. Let us first consider the special case $S=2$. There are then two (partially) massless points with corresponding dual operators having $\Delta_{\sigma}=3$ and $\Delta_{\sigma}=2$. The former is the ordinary graviton $\left(m^{2}=0\right)$ and the latter is the PM state that saturates the Higuchi bound $\left(m^{2}=2 H^{2}\right)$. We will discuss these two cases in turn.

- Graviton. - For a $\Delta_{\sigma}=3$ state, the exchange equation (2.13) becomes $\left(\Delta_{u}-\right.$ 2) $\hat{F}^{(0)}=\hat{C}_{0}$, with the simplest contact term on the right-hand side. Comparing this to the longitudinal coefficient function in (5.11), we find

$$
\hat{F}_{L}^{(2)}=\Delta_{u}\left(\Delta_{u}-2\right) \hat{F}^{(0)}=\hat{C}_{1} .
$$

Plugging this solution into (5.16), we obtain the corresponding bispectrum. ${ }^{10} \mathrm{We}$ wish to isolate the part of this result that can unambiguously be attributed to massless spin-2 exchange. In other words, we want to see whether any parts of this correlator can be mimicked by contact terms, and then subtract off these pieces.

The relevant contact contributions to the bispectrum are

$$
B_{c}=-\frac{\epsilon k_{3}^{3}}{3} \sum_{n} a_{n} U_{12}^{(0,0)} \hat{C}_{n}(u, 1)+\text { perms }
$$

To identify the parts that are degenerate with the PM-induced bispectrum, we consider the limit $k_{t} \equiv \sum_{a} k_{a} \rightarrow 0$. In this limit, the exchange bispectrum has a leading singularity scaling as $k_{t}^{-3}$. This singularity can be removed by adding the contact interaction $\hat{C}_{1}$, with $a_{1}=15$ in (5.21) (and all other coefficients zero). After this subtraction, the bispectrum still has a $k_{t}^{-1}$ singularity, which cannot be removed by another contact term. The part of the bispectrum that is cleanly associated with the exchange of a graviton therefore is

$$
B_{\mathrm{inf}}=3 \epsilon\left[\sum_{a \neq b} k_{a} k_{b}^{2}+\frac{8}{k_{t}} \sum_{a>b} k_{a}^{2} k_{b}^{2}-3 \sum_{a} k_{a}^{3}\right] .
$$

Up to a local term, $\sum_{a} k_{a}^{3}$, arising from the gauge transformation from spatially flat gauge to comoving gauge, this is precisely the famous bispectrum of single-field slow-roll inflation [80].

- PM graviton. - The exchange equation for a $\Delta_{\sigma}=2$ state is

$$
\Delta_{u} \hat{F}^{(0)}=\hat{C}_{0} .
$$

\footnotetext{
${ }^{10}$ In [1], a different exchange solution was utilized, with an unphysical contact term. The goal there was to mimic the number of derivatives of the bulk Lagrangian for graviton exchange. We see here that such a choice was unnecessary; one can just as well use a more physical exchange solution as a seed.
} 
Although the exchange solution is slightly different than for the graviton, after plugging it into (5.16), we still obtain a bispectrum with a $k_{t}^{-3}$ singularity. As before, we can subtract this singularity, together with the subleading $k_{t}^{-2}$ term, by adding a suitable choice of contact terms. In this case, we must take $a_{0}=24$ and $a_{1}=15$ in (5.21), with all the other coefficients vanishing.

This time, however, there is no leftover bispectrum! This means that the bispectrum due to the exchange of a PM graviton can be represented completely by a certain mixture of equilateral non-Gaussianity coming from inflaton self-interactions. This, however, does not rule out the possibility that inflaton correlators with PM exchange involve transcendental functions and therefore give shapes that are non-degenerate with contact diagrams. This is because our seed functions - the four-point functions of conformally coupled scalars with PM exchange - were rational functions.

Higher-spin exchange. The story becomes richer for higher spins, especially because there are now multiple PM points. Let us first describe a few qualitative features of these bispectra, before presenting details for the special cases $S=4$ and $6 .{ }^{11}$ Acting on the PM seed function $\hat{F}_{\Delta_{\sigma}}^{(0)}$ as in (5.18) yields the longitudinal part of the exchange correlator, which we then feed into (5.16) to produce the inflationary bispectrum. To isolate the part which is an unambiguous signature of PM exchange, we adopt the following procedure:

- The bispectrum will have a leading singularity for $k_{t} \rightarrow 0$ that can be removed by adding a contact interaction. In other words, part of the bispectrum shape is indistinguishable from equilateral non-Gaussianity. After removing those terms, the resulting shape will have a singularity scaling as $k_{t}^{1-S}$ (for any depth).

- The exchange of a PM field of depth $T=S-2$ can be absorbed completely by a sum of contact terms, with no exchange contribution remaining. ${ }^{12}$ These fields therefore do not produce a distinct bispectrum shape. Their imprint will only appear cleanly in the four-point function.

- After fixing the singularity for $k_{t} \rightarrow 0$, we still have the freedom to choose some contact terms to remove the least soft pieces of the bispectrum in the squeezed limit, so that

$$
\lim _{k_{3} \rightarrow 0}\left\langle\phi_{\vec{k}_{1}} \phi_{\vec{k}_{2}} \phi_{\vec{k}_{3}}\right\rangle \simeq \frac{1}{\left(k_{1} k_{3}\right)^{3}}\left(\frac{k_{3}}{k_{1}}\right)^{p} P_{J}(\cos \theta)+\cdots,
$$

where $\theta$ is the angle between $\vec{k}_{1}$ and $\vec{k}_{3}$, and the power $p$ depends on $S$ and $T$ in a nontrivial way. This subtraction can be done without changing the overall singularity in $k_{t}$. If there is any freedom left, we try to remove the highest possible Legendre polynomial $P_{J}$.

\footnotetext{
${ }^{11}$ Since we require a coupling to two identical $\Delta=2$ scalars in the relevant seed function, only particles of even spin can contribute to the bispectrum of an uncharged inflaton.

${ }^{12}$ We do not have a deep explanation for this fact, but it is interesting to speculate that it has something to do with the fact that the corresponding operator obeys a double-conservation condition [81-83], which somehow behaves differently from the other multiple-conservation conditions that dual PM operators satisfy.
} 
We now illustrate this procedure for PM fields of spin 4 and 6 .

- Spin-4: a spin-4 field has four PM points. Carrying out the procedure described above at each of them, we are able to pick various contact terms in (5.21) to isolate the parts of the inflationary bispectra due to the exchange of PM fields. We tabulate the necessary contact term coefficients and the resulting scalings in the squeezed limit (5.24) below:

\begin{tabular}{|cccccc|}
\hline$T$ & $a_{0}$ & $a_{1}$ & $a_{2}$ & $a_{3}$ & $(p, J)$ \\
\hline 0 & 0 & 0 & 2394 & 63 & $(2,4)$ \\
1 & 6880 & 9128 & 2178 & 63 & $(3,4)$ \\
2 & 4320 & 7128 & 1746 & 63 & - \\
3 & 2880 & 3708 & 1098 & 63 & $(3,0)$ \\
\hline
\end{tabular}

We see that there is a rich structure of scalings and angular dependences of the final inflationary bispectra. In particular, the depth-2 PM point has no unambiguous signature in the inflationary bispectrum. The other PM points lead to distinct behaviors in the squeezed limit.

- Spin-6: finally, we consider the exchange of a spin-6 field, with five PM points. A novelty of this situation is that, to perform the subtraction, we must introduce contact terms arising from integrating out intermediate spin- 2 particles:

$$
B_{c}=-\epsilon k_{3}^{3} \sum_{n} b_{n} P_{2}(\hat{\alpha}) U_{12}^{(2,0)}\left[\left(\Delta_{u}-2\right) \Delta_{u} \hat{C}_{n}(u, 1)\right]+\text { perms. }
$$

where $\hat{\alpha}=\left(k_{1}-k_{2}\right) / k_{3}$. The coefficients $a_{n}$ and $b_{n}$ in (5.21) and (5.25) are fixed by the same requirements as above. Their precise values are not very illuminating, so we don't display them. Instead, we present the final squeezed-limit scalings and angular dependences of the inflationary bispectra:

\begin{tabular}{|c|cccccc|}
\hline$T$ & 0 & 1 & 2 & 3 & 4 & 5 \\
\hline$(p, J)$ & $(2,6)$ & $(3,6)$ & $(4,6)$ & $(4,2)$ & - & $(4,2)$ \\
\hline
\end{tabular}

Again, this displays an interesting range of scalings, with the depth-4 point being degenerate with a set of contact interactions.

In summary, just like the famous graviton-induced bispectrum (5.22), PM fields generate new, distinct shapes of non-Gaussianity, which can be written as polynomials in the momenta divided by some overall power of the total energy $k_{t}$. These shapes uniquely characterize the presence of PM fields of various depths in the early universe. Whether consistent interacting theories of those PM fields (beyond gravity and gauge theory) exist remains an open problem (but see [84, 85]). 


\section{Conclusions}

In this paper, we have presented a highly streamlined and much more universal derivation of the spin-raising and weight-shifting operators that appeared in the original work on the cosmological bootstrap [1]. This was facilitated by the existence of a corresponding set of weight-shifting operators in conformal field theory [64, 66, 68], which when Fourier transformed can be applied in the cosmological context. Our treatment highlights the power and elegance of the bootstrap method by making manifest that a single seed function (corresponding to the correlator of conformally coupled scalars) can be transformed into all correlators of interest through the application of only two simple spin-raising and weightraising operators. This provides explicit results for inflationary bispectra arising from treelevel exchange of massive particles of arbitrary spin. The more systematic approach has also allowed us to characterize the effects from the exchange of (partially) massless fields of arbitrary spin, whose signatures have a rich structure with some surprising features.

While we have restricted our application of the weight-shifting technology to reproducing and generalizing the operators found in [1], many new applications have now opened up:

- It is straightforward to use our formalism to raise the spin of the external fields. In that case, the different tensor structures arise from the unique scalar seed because there is more than one way to raise the spin and weight of the external fields. These different ways lead to distinct answers which can be combined into the known tensor structures of spinning correlators.

- An interesting special case is correlators involving conserved tensors. For example, the stress tensor is dual to a bulk graviton and therefore part of any inflationary model. Correlation functions of conserved tensors a further constrained, because in addition to the conformal Ward identities discussed here, they must obey the WardTakahashi identities associated with current conservation. The interplay between these two differential constraints underlies the rich structure present for correlation functions of conserved operators. The weight-shifting formalism is a powerful way to study these correlation functions, and allows for a systematic classification similar to the one provided here for scalars.

- When the exchanged particles are massless, the spin-raised correlators are not guaranteed to be local. In flat space, the requirement of local four-point interactions is a powerful constraint on the space of consistent interactions between massless particles with spin [86, 87], making almost all theories, other than the familiar gauge theories and gravity, inconsistent. Similar considerations should restrict the theory space of viable interactions of massless fields in de Sitter space.

The tools that we have developed in this paper provide the first steps toward unraveling the intricate web of relations between these directions, and we will present our findings on these issues in a separate publication [57]. 


\section{Acknowledgments}

We are grateful to Hiroshi Isono, Denis Karateev, Petr Kravchuk, Toshifumi Noumi, Charlotte Sleight, Massimo Taronna, and Sasha Zhiboedov for discussions, and to David Simmons-Duffin for his patient tutoring in the weight-shifting formalism. We are also especially grateful to Nima Arkani-Hamed for encouragement, many fruitful discussions and collaboration. DB and CDP are supported by a Vidi grant of the Netherlands Organisation for Scientific Research (NWO) that is funded by the Dutch Ministry of Education, Culture and Science (OCW). AJ is supported in part by NASA grant NNX16AB27G. HL is supported by NSF grant AST-1813694 and DOE grant DE-SC0019018. GP is supported by the European Union's Horizon 2020 research and innovation programme under the MarieSklodowska Curie grant agreement number 751778. The work of DB and GP is part of the Delta-ITP consortium. DB, AJ, and GP thank the theory group at Harvard for its hospitality while this work was being completed. AJ thanks the Delta-ITP and the University of Amsterdam for hospitality while part of this work was completed.

\section{A Weight shifting in embedding space}

In the main text, we have utilized the spin-raising operator $\mathcal{S}_{12}$, cf. (4.10), and the weightraising operator $\mathcal{W}_{12}$, cf. (5.4). In this appendix, we wish to place these operators in a broader context and describe how they (and other useful operators) arise naturally from more formal conformal representation theory considerations. Our discussion is meant to be self-contained, although we do assume some familiarity with basic aspects of conformal field theory (see e.g. [69, 88]). For more details, readers are encouraged to consult [64].

\section{A.1 General preliminaries}

The general philosophy is fairly simple to state: given a solution to the conformal Ward identities (2.7) and (2.8), we would like to find differential operators that act on this solution to generate new solutions with different quantum numbers (either conformal weight or spin).

The most natural thing to look for would be a conformally-invariant operator that accomplishes this. Such operators do exist, but only in very special situations [89]. We can see this by considering the action of a putative operator, $\mathcal{D}$, on a conformal primary of weight $\Delta$ and spin $S$, which would be of the form

$$
\mathcal{D} O_{\Delta}^{(S)}=\tilde{O}_{\Delta^{\prime}}^{\left(S^{\prime}\right)}
$$

where the operator $\tilde{O}_{\Delta^{\prime}}^{\left(S^{\prime}\right)}$ transforms in some new representation of the conformal group, with weight $\Delta^{\prime}$ and spin $S^{\prime}$. Asking $\mathcal{D}$ to be conformally invariant is a strong constraint. First of all, it implies that the operator commutes with all conformal generators. In particular, this means that the operator is translationally invariant. It therefore cannot depend on coordinates and a differential operator with $n$ derivatives must then change the weight as follows

$$
\Delta \mapsto \Delta^{\prime}=\Delta+n
$$


Second, if the operator is conformally invariant it cannot change the quadratic Casimir eigenvalue of the representation, and therefore must satisfy

$$
\Delta(\Delta-d)+\mathcal{C}_{2}(S)=(\Delta+n)(\Delta+n-d)+\mathcal{C}_{2}\left(S^{\prime}\right),
$$

where we have substituted for $\Delta^{\prime}$ on the right-hand side and $\mathcal{C}_{2}(S)$ denotes the $\operatorname{SO}(d)$ quadratic Casimir of the spin- $S$ representation. ${ }^{13}$ Their precise values are not important; the point is that if we fix the spin representations that the operator $\mathcal{D}$ maps between, the constraint (A.3) becomes a linear equation for $\Delta$, which is only solved for one specific value. ${ }^{14}$ This means that - given a particular target weight and spin representation we are generically unable to find a conformally-invariant differential operator that maps us there from a given starting point.

The loophole to this argument is fairly intuitive, we must relax our requirements and search instead for differential operators that themselves transform in some representation of the conformal group. We therefore are in search of conformally-covariant (as opposed to invariant) differential operators. The construction of such weight-shifting operators was performed systematically in [64], and here we wish to review their construction. Special cases of weight-shifting operators were constructed earlier in [63].

\section{A.2 Some group theory}

Some representation-theoretic considerations are helpful in order to understand what weight-shifting operators should exist. These details are not essential and readers who are willing to take the existence and transformation properties of weight-shifting operators on faith can skip to the next subsection to see them constructed explicitly.

It is useful to consider conformally-covariant differential operators that transform in finite-dimensional representations of the conformal algebra. ${ }^{15}$ The content of these finitedimensional representations, $W$, can be understood by thinking of them as analytically continued $\mathrm{SO}(d+2)$ representations; they decompose under dilations $\times \mathrm{SO}(d)$ as a direct sum

$$
W=\bigoplus_{i=-j}^{j} W_{i},
$$

where the dilation eigenvalue of the subspaces $W_{i}$ runs from $-j$ to $j$ and is analogous to the spin quantum number, $J_{z}$, for $\mathrm{SO}(3)$ representations.

We now consider the tensor product of $W$ with another irreducible conformal representation, which we denote by $V_{\Delta, S}$, where $\Delta, S$ label the weight and spin of the lowest-weight

\footnotetext{
${ }^{13}$ More generally, any $\mathrm{SO}(d)$ representations can appear in (A.1). This does not change the argument. In what follows, we will often restrict to spin- $S$ representations for notational simplicity, but nothing depends on this choice.

${ }^{14}$ An example is that $\partial_{\mu} J^{\mu}$ transforms like a scalar, but only for $\Delta_{J}=d-1$.

${ }^{15}$ Note that these finite-dimensional representations are not unitary — as they represent a non-compact algebra - but this does not affect their usefulness. These representations should be thought of as a tool to generate operators with the kinematic transformation properties we desire. Imposing unitarity of the final results will require additional information beyond kinematics that will have to be input at a later time in some situations.
} 
state. The goal is to decompose the tensor product $W \otimes V_{\Delta, S}$ into irreducible representations of the conformal group. This is equivalent to finding the conformal primary operators that appear in the product representation. A straightforward way to accomplish this is to use the state-operator correspondence and think of the representation $W$ as being generated by the conformal primary operator $w_{-j}^{\left(S_{w}\right)}(0)$ with weight $\Delta_{w}=-j$ and spin $S_{w}$ and think of the representation $V_{\Delta, S}$ as being generated by the conformal primary $O_{\Delta}^{(S)}(0)$. Since $W$ is finite-dimensional, $w_{-j}^{\left(S_{w}\right)}(0)$ will have a finite number of descendants - we can only take so many derivatives before the resulting states are guaranteed to be null.

Primary operators appearing in $W \otimes V_{\Delta, S}$ can be constructed by considering products of $w$ and $O$ of the form [64]

$$
\tilde{O}_{\Delta^{\prime}}^{S^{\prime}}=\partial_{i_{1}} \cdots \partial_{i_{m}} w_{-j}^{\left(S_{w}\right)}(0) \otimes O_{\Delta}^{(S)}(0)+c_{1} \partial_{i_{1}} \cdots \partial_{i_{m-1}} w_{-j}^{\left(S_{w}\right)}(0) \otimes \partial_{i_{m}} O_{\Delta}^{(S)}(0)+\cdots,
$$

where $m$ can range from 0 to $2 j$ and the ellipses denote all other ways of distributing the $m$ derivatives on both operators. The various coefficients can be fixed uniquely by demanding that the expression (A.5) is a primary operator, i.e. that it is annihilated by the special conformal generator. We are being somewhat schematic about the spin representations, but the spin representations appearing in (A.5) should also be decomposed into irreducible components. Note that this involves both the indices carried by the derivatives as well as possible $\mathrm{SO}(d)$ indices of the operators $w$ and $O$.

Each of the resulting primary operators constructed in this way will have weight $\Delta^{\prime}=\Delta-j+m$ and will transform in some definite $\mathrm{SO}(d)$ representation. Once we have decomposed (A.5) like this, we can interpret the result as a differential operator acting on $O_{\Delta}^{(S)}$ by recalling that $w$ transforms in a finite-dimensional representation of the conformal group, which therefore has a finite basis, $e^{A}$. We can write (A.5) as

$$
\begin{aligned}
\tilde{O}_{\Delta^{\prime}}^{S^{\prime}} & =e^{A} \otimes\left(\partial_{i_{1}} \cdots \partial_{i_{m}} w_{A}(0) \otimes O_{\Delta}^{(S)}(0)+c_{1} \partial_{i_{1}} \cdots \partial_{i_{m-1}} w_{A}(0) \otimes \partial_{i_{m}} O_{\Delta}^{(S)}(0)+\cdots\right) \\
& \equiv e^{A} \otimes \mathcal{D}_{A} O_{\Delta}^{(S)} .
\end{aligned}
$$

The operator $\mathcal{D}_{A}$ is a weight-shifting operator: it changes the conformal weight and/or spin representation of the operator $O$ and transforms in a finite-dimensional representation of the conformal group.

The preceding discussion was very abstract, so we now proceed to construct some particularly useful weight-shifting operators explicitly. It should be noted, however, that there are, in principle, an infinite number of such operators. Any finite-dimensional representation of the conformal group can be used to construct a set of weight-shifting operators. It could be that some more exotic possibilities are also of use in cosmology.

\section{A.3 Vector representation}

One of the most important sets of weight-shifting operators arises from the finitedimensional vector representation of the conformal group, $W_{M}$ (which has $j=1$ ), where $M$ is an embedding space index with $d+2$ components. Decomposing this $\mathrm{SO}(d+1,1)$ 
representation into $\mathrm{SO}(1,1) \times \mathrm{SO}(d)$ representations as in (A.4), we find

$$
\square \underset{\mathrm{SO}(1,1) \times \mathrm{SO}(d)}{\longrightarrow}(\bullet)_{-1} \oplus(\square)_{0} \oplus(\bullet)_{1},
$$

where $\bullet$ denotes the trivial spin representation, and the subscripts are the dilation weight. ${ }^{16}$ This is just a pictorial way of representing the information that a $d+1+1$ split of the vector $W_{M}$ contains two scalars, $W_{-1}$ and $W_{0}$, and one $d$-dimensional vector, $W_{i}$. Equivalently, the lowest-weight state in the representation is a scalar, $w_{-1}(0)$, with weight $\Delta_{w}=-1$. This scalar satisfies the differential equation

$$
\partial_{(i} \partial_{j)_{T}} w_{-1}(0)=0
$$

where the notation $(\cdots)_{T}$ denotes the symmetric trace-free part.

By considering products of these operators with a primary of interest, like in (A.6), we can see what kind of weight-shifting operators they correspond to.

- Weight -1 : the lowest-weight state has weight $\Delta=-1$. The corresponding weightshifting operator will shift the weight of the representation by $\Delta \mapsto \Delta-1$. This state is a scalar under $\mathrm{SO}(d)$, so it does not change the spin representation.

- Weight 0: the first descendent state transforms as a vector under $\mathrm{SO}(d)$ and has weight $\Delta=0$. The weight-shifting operators associated with this state leave the weight invariant, but change the spin representation. To see how the spin for example of a spin- $S$ representation is changed, we decompose the tensor product

$$
S^{T} \otimes \square=S_{S-1}^{T} \oplus S_{S+1}^{T} \oplus \square^{T} .
$$

Acting on spin- $S$ representations there are therefore three weight-shifting operators: one which shifts the spin down by one unit, one that shifts the spin up by one unit, and a third operator that projects onto a mixed-symmetry representation. ${ }^{17}$ (For more general spin representations the story is similar, but the details can be more complicated.)

- Weight 1: finally, the highest-weight state is a scalar with weight $\Delta=1$ and the corresponding weight-shifting operator will shift the weight of the representation by $\Delta \mapsto \Delta+1$.

Operators in embedding space. The vector representation therefore gives rise to a set of differential operators that shift the dimension and spin of CFT operators $O(X, Z)$, which we denote by

$$
\mathcal{D}_{M}^{\alpha \beta}:[\Delta, S] \mapsto[\Delta+\alpha, S+\beta] .
$$

\footnotetext{
${ }^{16}$ The dilation weights of the various operators can be obtained by noting that the representation must shorten at the second level of descendents, with the spin-2 state becoming null. For a discussion of the shortening of conformal representations, see [90].

${ }^{17}$ We will not utilize weight-shifting operators that generate mixed-symmetry representations, because our principal interest is in $d=3$ dimensions, where mixed-symmetry representations can all be dualized to symmetric tensor representations. However, these operators may be useful for some calculations.
} 
In principle, these operators can be constructed by solving the differential equation (A.8) and then building states of the form (A.6). However, in practice, the formal representationtheoretic arguments we have just reviewed are more useful to catalog which weight-shifting operators should exist, and then construct them directly in embedding space.

The benefit of working in embedding space is that it is easy to implement the correct conformal transformation properties of the weight-shifting operators. For example, weight-shifting operators coming from the vector representation will carry an uncontracted embedding space index. It is then algorithmic to construct weight-shifting operators: the above list completely catalogs the possible operators, our task is to find embedding space expressions with the correct conformal dimensions and spin weights.

One additional subtlety needs to be addressed: we must ensure that the resulting expressions preserve the Euclidean section of the projective lightcone. This is not automatic because embedding space tensors are invariant under the shift $Z \mapsto Z+\beta X$ and subject to the constraints

$$
X^{2}=X \cdot Z=Z^{2}=0 .
$$

We must then make sure that these combined constraints are preserved by the differential operators we construct. This turns out to be a strong enough requirement to uniquely fix the embedding space expressions for each weight-shifting operator.

The weight-shifting operators in the vector representation were constructed explicitly in appendix $\mathrm{C}$ of [64]. Here, we summarize their results. The algorithm involves first making an ansatz that has the correct weights: ${ }^{18}$

$$
\begin{aligned}
\mathcal{D}_{M}^{-0}= & X_{M} \\
\mathcal{D}_{M}^{0-}= & a_{1} \frac{\partial}{\partial Z^{M}}+a_{2} Z_{M} \frac{\partial^{2}}{\partial Z^{2}}+a_{3} X_{M} \frac{\partial^{2}}{\partial X \cdot \partial Z}+a_{4} X_{M} Z \cdot \frac{\partial}{\partial X} \frac{\partial^{2}}{\partial Z^{2}}, \\
\mathcal{D}_{M}^{0+}= & b_{1} Z_{M}+b_{2} X_{M} Z \cdot \frac{\partial}{\partial X}, \\
\mathcal{D}_{M}^{+0}= & c_{1} \frac{\partial}{\partial X^{M}}+c_{2} X_{M} \frac{\partial^{2}}{\partial X^{2}}+c_{3} Z_{M} \frac{\partial^{2}}{\partial Z \cdot \partial X}+c_{4} Z \cdot \frac{\partial}{\partial X} \frac{\partial}{\partial Z^{M}} \\
& +c_{5} X_{M} Z \cdot \frac{\partial}{\partial X} \frac{\partial^{2}}{\partial Z \cdot \partial X}+c_{6} Z_{M} Z \cdot \frac{\partial}{\partial X} \frac{\partial^{2}}{\partial Z^{2}}+c_{7} X_{M}\left(Z \cdot \frac{\partial}{\partial X}\right)^{2} \frac{\partial^{2}}{\partial Z^{2}} .
\end{aligned}
$$

We now wish to fix the various coefficients in the operators above. First, we require that the action of the weight-shifting operators preserves invariance under the shift $Z \mapsto Z+\beta X$. This implies

$$
\left(X \cdot \frac{\partial}{\partial Z}\right) \mathcal{D}_{M} f_{\Delta, S}(X, Z)=0
$$

where $f_{\Delta, S}$ is any homogeneous polynomial of spin $S$ and weight $\Delta$ that is invariant under the shift of $Z[64,68]$. The latter can be written as

$$
f_{\Delta, S}(X, Z)=(X \cdot Y)^{-\Delta-S}\left(P^{M} Q^{N} C_{M N}\right)^{S},
$$

\footnotetext{
${ }^{18}$ Note that $Z$ raises the spin by one, $X$ lowers the weight by one and derivatives do the opposite. The action of the operators $X \cdot \partial_{X}=-\Delta$ and $Z \cdot \partial_{Z}=S$ can be absorbed into the constants, while $X \cdot \partial_{Z}=0$ is one of the constraints we will impose, so this operator does not appear.
} 
where $C_{M N}=Z_{M} X_{N}-Z_{N} X_{M}$ and $Y, P, Q$ are arbitrary constant vectors. Second, we also have to ensure that the constraints (A.11) are preserved. This is somewhat tricker to enforce because there are many possible ways to extend the operators away from the lightcone. In practice, we only need to impose this constraint on a small number of polynomials in order to completely fix all of the free coefficients. The following set of polynomials, which all have weight $\Delta$ and spin $S$, does the job [64]

$$
\begin{aligned}
& g_{1}(X, Z)=X^{2} f_{\Delta+2, S}(X, Z), \\
& g_{2}(X, Z)=S^{M} X^{N} C_{M N} f_{\Delta+2, S-1}(X, Z), \\
& g_{3}(X, Z)=Z^{M} X^{N} C_{M N} f_{\Delta+2, S-2}(X, Z), \\
& g_{4}(X, Z)=S^{M} S_{N} C_{M O} C^{O N} f_{\Delta+2, S-2}(X, Z),
\end{aligned}
$$

where $S$ is an arbitrary constant vector. It is straightforward to check that these polynomials all vanish after imposing (A.11). Finally, we act with the weight-shifting operators before imposing the constraints, then impose the constraints and demand that the result vanishes. This completely fixes all the remaining free coefficients and we find

$$
\begin{aligned}
\mathcal{D}_{M}^{-0}= & X_{M}, \\
\mathcal{D}_{M}^{0-}= & \left((\Delta-d+2-S) \delta_{M}^{N}+X_{M} \frac{\partial}{\partial X_{N}}\right)\left((d-4+2 S) \frac{\partial}{\partial Z^{N}}-Z_{N} \frac{\partial^{2}}{\partial Z^{2}}\right), \\
\mathcal{D}_{M}^{0+}= & (S+\Delta) Z_{M}+X_{M} Z \cdot \frac{\partial}{\partial X}, \\
\mathcal{D}_{M}^{+0}= & c_{1} \frac{\partial}{\partial X^{M}}+c_{2} X_{M} \frac{\partial^{2}}{\partial X^{2}}+c_{3} Z_{M} \frac{\partial^{2}}{\partial Z \cdot \partial X}+c_{4} Z \cdot \frac{\partial}{\partial X} \frac{\partial}{\partial Z^{M}} \\
& +c_{5} X_{M} Z \cdot \frac{\partial}{\partial X} \frac{\partial^{2}}{\partial Z \cdot \partial X}+c_{6} Z_{M} Z \cdot \frac{\partial}{\partial X} \frac{\partial^{2}}{\partial Z^{2}}+c_{7} X_{M}\left(Z \cdot \frac{\partial}{\partial X}\right)^{2} \frac{\partial^{2}}{\partial Z^{2}},
\end{aligned}
$$

where the coefficients $c_{n}$ in (A.25) are given by

$$
\begin{array}{rlrl}
c_{1} & =\left(\frac{d}{2}-\Delta-1\right)(\Delta+S-1)(d-\Delta+S-2), & c_{5} & =\frac{d}{2}+S-2, \\
c_{2} & =-\frac{1}{2}(\Delta+S-1)(d-\Delta+S-2), & c_{6} & =\frac{d}{2}-\Delta-1, \\
c_{3} & =-\left(\frac{d}{2}-\Delta-1\right)(\Delta+S-2), & c_{7} & =-\frac{1}{2} . \\
c_{4} & =-\left(\frac{d}{2}-\Delta-1\right)(d-\Delta+S-2), &
\end{array}
$$

The operators defined in this way preserve the Euclidean section of the projective lightcone and shift the weights of the operators they act on (as indicated by the superscripts of $\mathcal{D}_{M}^{\alpha \beta}$ ).

Bi-local operators. The weight-shifting operators constructed in this way satisfy all of our requirements - they are conformally covariant and change the quantum numbers of representations - but it is natural to ask if we can do better. It would be preferable to have some objects that are actually conformally invariant. From the arguments in section A.1, we don't expect to be able to accomplish this with operators that act at a single point, but 
nothing prevents us from combining pairs of weight-shifting operators into singlets that are bi-local. In particular, it is useful to introduce the following combinations of operators that act at two points (labeled by 1 and 2) by contracting their embedding space indices

$$
\begin{aligned}
\mathcal{S}_{12}^{++} & \equiv \mathcal{D}_{1}^{0+} \cdot \mathcal{D}_{2}^{0+}, \\
\mathcal{S}_{12}^{--} & \equiv \mathcal{D}_{1}^{0-} \cdot \mathcal{D}_{2}^{0-}, \\
\mathcal{W}_{12}^{++} & \equiv \mathcal{D}_{1}^{+0} \cdot \mathcal{D}_{2}^{+0}, \\
\mathcal{W}_{12}^{--} & \equiv \mathcal{D}_{1}^{-0} \cdot \mathcal{D}_{2}^{-0}
\end{aligned}
$$

Defined in this way, the operators $\mathcal{S}_{12}^{ \pm \pm}$raise (lower) the spins at points 1 and 2, while the operators $\mathcal{W}_{12}^{ \pm \pm}$raise (lower) the dimensions. In the main text, we have used the operator $\mathcal{W}_{12}^{++} \equiv \mathcal{W}_{12}$ to raise the weight of external fields from $\Delta=2$ to $\Delta=3$.

The operators $\mathcal{S}_{12}^{ \pm \pm}$and $\mathcal{W}_{12}^{ \pm \pm}$act in the same way at both points, but nothing requires us to combine the operators in this way. In fact, an extremely useful combination is the operator

$$
\begin{aligned}
D_{12} \equiv \mathcal{D}_{1}^{0+} \cdot \mathcal{D}_{2}^{-0} & =\left(\Delta_{1}+S_{1}\right) Z_{1} \cdot X_{2}+\left(X_{1} \cdot X_{2}\right) Z_{1} \cdot \frac{\partial}{\partial X_{1}} \\
& =-\left(Z_{1} \cdot X_{2}\right) X_{a} \cdot \frac{\partial}{\partial X_{1}}+\left(Z_{1} \cdot X_{2}\right) Z_{1} \cdot \frac{\partial}{\partial Z_{1}}+\left(X_{1} \cdot X_{2}\right) Z_{1} \cdot \frac{\partial}{\partial X_{1}},
\end{aligned}
$$

which raises the spin by one unit at point 1 and lowers the dimension by one at point 2 . This operator was first derived in [63]. They also constructed an operator that raises the spin and lowers the weight at the same point, but to construct this operator we have to consider the adjoint representation, which we do next.

\section{A.4 Adjoint representation}

We can repeat the same construction with any finite-dimensional representation of the conformal group. Another particular useful example is the adjoint representation, which is the anti-symmetric tensor representation of $\mathrm{SO}(d+1,1)$; under $\mathrm{SO}(1,1) \times \mathrm{SO}(d)$, it decomposes as

$$
\square \underset{\mathrm{SO}(1,1) \times \operatorname{SO}(d)}{\longrightarrow}(\square)_{-1} \oplus(\bullet \oplus \square)_{0} \oplus(\square)_{1}
$$

We see that the adjoint representation has $j=-1$ and is generated by a vector primary state, $w_{-1}^{i}(0)$. In this case we can again employ representation-theoretic arguments to count the weight-shifting operators that should exist. We will not be quite as systematic as we were for the vector representation, but rather we will just construct some useful operators.

- Weight -1 : the lowest-weight state is a vector, so the degenerate primary, $w^{i}$, carries a $\mathrm{SO}(d)$ vector index. Acting on a symmetric tensor representation, the spin decomposition is the same as in (A.9) and we should be able to find weight-shifting operators that lower the weight by one unit and either raise or lower the spin by one unit. In addition, there is an operator that projects onto a mixed-symmetry representation, but we will not utilize it. 
- Weight 0: at the first level of descendants there are two kinds of weight-shifting operators. The first kind is associated to the trivial spin representation and does nothing to either the spin or the conformal weight. These operators are actually exactly the conformal generators: they are conformally-covariant differential operators that transform in the adjoint and keep us in the same representation. The second kind of weight-shifting operator at this level doesn't change the conformal dimension, but can map us to spin representations appearing in the decomposition

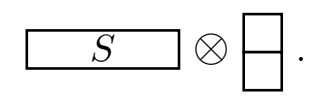

We will not use these operators because only mixed-symmetry tensors appear in this decomposition.

- Weight 1: the story at weight 1 is similar to the case at weight -1 : there are three kinds of operators. Two of them raise the dimension by one unit and either spin-up or spin-down a symmetric tensor operator. The third operator again maps a symmetric tensor to a mixed-symmetry representation.

Operators in embedding space. Now that we know what type of weight-shifting operators should exist, we can search for them in embedding space like we did for the vector representation. The difference is that in this case the operators will carry a pair of antisymmetric embedding space indices, as they must transform in the adjoint. In practice, this means that we can construct the adjoint embedding space operators by multiplying and anti-symmetrizing the vector embedding space operators.

Rather than systematically derive all possible operators, like we did for the vector representation, we instead only quote some operators which are particularly useful. For example, the operator that lowers the weight by one and raises the spin by one is

$$
\mathcal{D}_{M N}^{-+}=X_{[M} Z_{N]}
$$

Another semi-trivial example is provided by the conformal generators

$$
\mathcal{D}_{M N}^{00}=J_{M N}=X_{[M} \frac{\partial}{\partial X^{N]}}+Z_{[M} \frac{\partial}{\partial Z^{N]}},
$$

which by itself do not do anything to correlation functions if summed over all points, but can be useful in tandem with one of the other weight-shifting operators.

There are three other weight-shifting operators that can be built from the adjoint representation, which we do not construct explicitly because they are not needed in our analysis. The operators act in the following way: one operator lowers both the weight and spin at a point by one unit, one operator raises both the weight and spin at a point by one unit and finally another operator raises the weight by one unit and lowers the spin by one unit. If desired, these operators can be constructed by antisymmetrizing various combinations of the operators in the vector representation. 
Bi-local operators. As before, these operators are most useful when paired together into conformally-invariant combinations. For example, a convenient combination is

$$
H_{12} \equiv \mathcal{D}_{1}^{-+} \cdot \mathcal{D}_{2}^{-+}=\left(Z_{1} \cdot Z_{2}\right)\left(X_{1} \cdot X_{2}\right)-\left(Z_{1} \cdot X_{2}\right)\left(Z_{2} \cdot X_{1}\right),
$$

which lowers the weight and raises the spin by one unit at both points 1 and 2. Another very useful operator is

$$
\begin{aligned}
D_{11} & \equiv \mathcal{D}_{1}^{-+} \cdot J_{2} \\
& =\left(X_{1} \cdot X_{2}\right) Z_{1} \cdot \frac{\partial}{\partial X_{2}}-\left(X_{2} \cdot Z_{1}\right) X_{1} \cdot \frac{\partial}{\partial X_{2}}+\left(X_{1} \cdot Z_{2}\right) Z_{1} \cdot \frac{\partial}{\partial Z_{2}}-\left(Z_{2} \cdot Z_{1}\right) X_{1} \cdot \frac{\partial}{\partial Z_{2}},
\end{aligned}
$$

which raises the spin and lowers the weight by one unit at point 1 , but does nothing at point 2. This operator was also considered in [63].

\section{B Weight shifting in Fourier space}

Cosmological correlation functions naturally live in Fourier space, so we would like to understand the action of the bi-local weight-shifting operators in Fourier space. This involves first projecting the weight-shifting operators from embedding space to position space, and then transforming the result to Fourier space. In this appendix, we will give details of the weight-shifting operators in Fourier space. We will present results that are valid for general dimensions.

\section{B.1 Projection to position space}

We first consider projecting the embedding space weight-shifting operators to the Euclidean section of the lightcone. Using $X^{M}=\left(1, x^{2}, x^{i}\right)$ and $Z^{M}=\left(0,2 \vec{x} \cdot \vec{z}, z^{i}\right)$, we can write the derivatives with respect to the embedding coordinates as

$$
\begin{aligned}
\frac{\partial}{\partial X^{M}} & =\frac{\partial x^{i}}{\partial X^{M}} \frac{\partial}{\partial x^{i}}+\frac{\partial z^{i}}{\partial X^{M}} \frac{\partial}{\partial z^{i}}=\left(-\Delta-x^{j} \frac{\partial}{\partial x^{j}}, 0, \frac{\partial}{\partial x^{i}}\right) \\
\frac{\partial}{\partial Z^{M}} & =\frac{\partial x^{i}}{\partial Z^{M}} \frac{\partial}{\partial x^{i}}+\frac{\partial z^{i}}{\partial Z^{M}} \frac{\partial}{\partial z^{i}}=\left(-x^{j} \frac{\partial}{\partial z^{j}}, 0, \frac{\partial}{\partial z^{i}}\right) .
\end{aligned}
$$

This allows us to write all relevant scalar products in embedding space and scalar products in position space:

$$
\begin{array}{rlrl}
Z_{a} \cdot Z_{b} & =\vec{z}_{a} \cdot \vec{z}_{b}, & Z_{a} \cdot \frac{\partial}{\partial Z_{b}}=\vec{z}_{a} \cdot \frac{\partial}{\partial \vec{z}_{b}}, \\
X_{a} \cdot Z_{b} & =\left(\vec{x}_{a}-\vec{x}_{b}\right) \cdot \vec{z}_{b}, & Z_{a} \cdot \frac{\partial}{\partial X_{b}}=\vec{z}_{a} \cdot \frac{\partial}{\partial \vec{x}_{b}}, \\
X_{a} \cdot X_{b} & =-\frac{\left(x_{a}-x_{b}\right)^{2}}{2}, & X_{a} \cdot \frac{\partial}{\partial X_{b}}=-\Delta_{b}+\left(\vec{x}_{a}-\vec{x}_{b}\right) \cdot \frac{\partial}{\partial \vec{x}_{b}}, \\
\frac{\partial}{\partial Z_{a}} \cdot \frac{\partial}{\partial Z_{b}}=\frac{\partial}{\partial \vec{z}_{a}} \cdot \frac{\partial}{\partial \vec{z}_{b}}, & X_{a} \cdot \frac{\partial}{\partial Z_{b}}=\left(\vec{x}_{a}-\vec{x}_{b}\right) \cdot \frac{\partial}{\partial \vec{z}_{b}}, \\
\frac{\partial}{\partial X_{a}} \cdot \frac{\partial}{\partial X_{b}}=\frac{\partial}{\partial \vec{x}_{a}} \cdot \frac{\partial}{\partial \vec{x}_{b}}, &
\end{array}
$$


where $a, b$ label different positions. We will not write out the full position space expressions for the weight-shifting operators, but they can be obtained straightforwardly by substituting these scalar products into the embedding space expressions given in appendix A.

\section{B.2 Fourier-transformed operators}

Once the weight-shifting operators have been written in position space, it is an algorithmic (though tedious!) task to transform these operators to Fourier space. In the following, we record the Fourier space expressions for a variety of useful weight-shifting operators that act on pairs of points.

- The operator $\mathcal{W}_{12}^{--}$, defined in (A.30), lowers weights at points 1 and 2 by one unit. In Fourier space, it takes the form

$$
\mathcal{W}_{12}^{--}=\frac{1}{2}\left(\frac{\partial}{\partial \vec{k}_{1}}-\frac{\partial}{\partial \vec{k}_{2}}\right) \cdot\left(\frac{\partial}{\partial \vec{k}_{1}}-\frac{\partial}{\partial \vec{k}_{2}}\right) \equiv \frac{1}{2} \vec{K}_{12} \cdot \vec{K}_{12},
$$

where we have defined the differential operator

$$
\vec{K}_{12} \equiv \frac{\partial}{\partial \vec{k}_{1}}-\frac{\partial}{\partial \vec{k}_{2}}
$$

- Similarly, the operator $\mathcal{W}_{12}^{++}$in (A.29) raises the weights by one at both points 1 and 2. It was used in section 5.1 to change the weight of the external fields from $\Delta=2$ to $\Delta=3$. In general, this operator takes a very complicated form in Fourier space,

$$
\begin{aligned}
\mathcal{W}_{12}^{++} \propto & D_{1}^{(A)} D_{2}^{(A)} \mathcal{W}_{12}^{--}-\left(\vec{k}_{1} \cdot \vec{z}_{1}\right)\left(\vec{k}_{2} \cdot \vec{z}_{2}\right)\left(\delta_{1} \delta_{2}\left(\vec{z}_{1} \cdot \vec{z}_{2}\right) \nabla_{\vec{z}_{1}}^{2} \nabla_{\vec{z}_{2}}^{2}+\eta_{12}^{2} \eta_{21}^{2}\left(\partial_{\vec{z}_{1}} \cdot \partial_{\vec{z}_{2}}\right)\right) \\
& +\left[\delta_{1} D_{2}^{(a)}\left(\vec{K}_{12} \cdot \vec{z}_{1}\right)\left(\left(\vec{k}_{1} \cdot \vec{z}_{1}\right) \nabla_{\vec{z}_{1}}^{2}-\tau_{1}\left(\vec{k}_{1} \cdot \partial_{\vec{z}_{1}}\right)\right)-\eta_{11}^{2}\left(\vec{k}_{1} \cdot \vec{z}_{1}\right) D_{1}^{(A)}\left(\vec{K}_{12} \cdot \partial_{\vec{z}_{1}}\right)\right. \\
& +\delta_{2}\left(\vec{k}_{1} \cdot \vec{z}_{1}\right)\left(D_{21}^{(B)}+\left(\vec{k}_{1} \cdot \vec{z}_{1}\right)\left(\sigma_{2}\left(1+\Delta_{2}-d\right)-\tau_{2}\left(\vec{z}_{2} \cdot \partial_{\vec{z}_{2}}\right)\right)\right) \nabla_{\vec{z}_{1}}^{2}-\delta_{1} \eta_{12} \sigma_{2}\left(\vec{k}_{1} \cdot \vec{z}_{1}\right) \\
& -\delta_{2}\left[\sigma_{2}\left(-4+d+2 S_{1}\right)\left(1-d+\Delta_{2}\right)\left(\vec{k}_{1} \cdot \vec{z}_{1}\right)+\tau_{1} D_{21}^{(B)}\right]\left(\vec{k}_{1} \cdot \partial_{\vec{z}_{1}}\right) \\
& +(1 \leftrightarrow 2)]
\end{aligned}
$$

where we have defined

$$
\begin{aligned}
& D_{a}^{(A)} \equiv\left(\vec{k}_{a} \cdot \vec{z}_{a}\right)^{2} \nabla_{\vec{z}_{1}}^{2}-\left(d-4+2 S_{a}\right)\left(\vec{k}_{a} \cdot \vec{z}_{a}\right)\left(\vec{k}_{a} \cdot \partial_{\vec{z}_{a}}\right)-k_{a}^{2} \sigma_{a}^{2}, \\
& D_{a b}^{(B)} \equiv \delta_{a}\left(\tau_{a}\left(\vec{z}_{1} \cdot \vec{z}_{2}\right)\left(\vec{k}_{a} \cdot \partial_{\vec{z}_{a}}\right)+\sigma_{b}\left(\vec{k}_{a} \cdot \vec{z}_{b}\right)\right)+\eta_{a b}\left(\vec{k}_{a} \cdot \vec{z}_{a}\right)\left(\vec{z}_{b} \cdot \partial_{\vec{z}_{a}}\right),
\end{aligned}
$$

and

$$
\begin{aligned}
\sigma_{a}^{2} & \equiv\left(\Delta_{a}+S_{a}-1\right)\left(d-2-\Delta_{a}+S_{a}\right), \\
\eta_{a b}^{2} & \equiv\left(d-3-\Delta_{a}+S_{a}\right)\left(d-2 \Delta_{b}\right), \\
\tau_{a} & \equiv \Delta_{a}+S_{a}-1, \\
\delta_{a} & \equiv d-\Delta_{a} .
\end{aligned}
$$

Fortunately, this becomes somewhat more manageable when acting on scalars:

$$
\begin{aligned}
\mathcal{W}_{12}^{++}= & \left(k_{1} k_{2}\right)^{2} \mathcal{W}_{12}^{--}-\left(d-2 \Delta_{1}\right)\left(d-2 \Delta_{2}\right) \vec{k}_{1} \cdot \vec{k}_{2} \\
& +\left(k_{2}^{2}\left(d-2 \Delta_{1}\right)\left(d-1-\Delta_{1}+\vec{k}_{1} \cdot \vec{K}_{12}\right)+(1 \leftrightarrow 2)\right) .
\end{aligned}
$$


In the main text, this operator played an important role and was denoted $\mathcal{W}_{12}$, i.e. we dropped the superscripts to avoid clutter.

- The spin-raising operator $\mathcal{S}_{12}^{++}$, defined in (A.27), has the following Fourier representation

$$
\begin{aligned}
\mathcal{S}_{12}^{++}= & \left(S_{1}+\Delta_{1}-1\right)\left(S_{2}+\Delta_{2}-1\right) \vec{z}_{1} \cdot \vec{z}_{2}-\left(\vec{z}_{1} \cdot \vec{k}_{1}\right)\left(\vec{z}_{2} \cdot \vec{k}_{2}\right) \mathcal{W}_{12}^{--} \\
& +\left[\left(S_{1}+\Delta_{1}-1\right)\left(\vec{k}_{2} \cdot \vec{z}_{2}\right)\left(\vec{z}_{1} \cdot \vec{K}_{12}\right)+(1 \leftrightarrow 2)\right] .
\end{aligned}
$$

This operator raises the spin by one unit at both points 1 and 2 , which is useful for generating spinning correlators [57].

- The operator $D_{12}$, defined in (A.31), raises the spin at point 1 and lowers the weight at point 2. In Fourier space, it reads

$$
D_{12}=\left(\Delta_{1}+S_{1}-1\right) \vec{z}_{1} \cdot \vec{K}_{12}-\left(\vec{z}_{1} \cdot \vec{k}_{1}\right) \mathcal{W}_{12}^{--},
$$

where we have dropped an overall factor of $i$. We have used this operator in the main text to raise the spin of exchanged fields.

- The operator $D_{11}$ in (A.37) both raises the spin and lowers the weight at the point 1 and is useful for raising the spin of external fields [57]. In Fourier space, it becomes

$$
D_{11}=\left(\Delta_{2}-d+\vec{k}_{2} \cdot \vec{K}_{12}\right) \vec{z}_{1} \cdot \vec{K}_{12}-\left(\vec{k}_{2} \cdot \vec{z}_{1}\right) \mathcal{W}_{12}^{--}-\vec{z}_{2} \cdot \vec{K}_{12} \vec{z}_{1} \cdot \partial_{\vec{z}_{2}}+\left(\vec{z}_{1} \cdot \vec{z}_{2}\right) \partial_{\vec{z}_{2}} \cdot \vec{K}_{12},
$$

where we have again dropped an overall factor of $i$.

- It is often helpful to use the operator $H_{12}$, defined in (A.36), which raises the spin and lowers the weight by one unit at both points 1 and 2 . Its Fourier representation is

$$
H_{12}=2 \vec{z}_{1} \cdot \vec{K}_{12} \vec{z}_{2} \cdot \vec{K}_{12}-\left(\vec{z}_{1} \cdot \vec{z}_{2}\right) \vec{K}_{12} \cdot \vec{K}_{12} \cdot
$$

- Although we have not made use of it in this work, for completeness let us also give the expression for the spin-lowering operator $\mathcal{S}_{12}^{--}$, defined in (A.28), which lowers the spin by one unit at both points 1 and 2 :

$$
\begin{aligned}
\mathcal{S}_{12}^{--}= & D_{1}^{(C)} D_{2}^{(C)} \mathcal{W}_{12}^{--}-\rho_{1} \rho_{2}\left(\vec{z}_{1} \cdot \vec{z}_{2}\right) \nabla_{\vec{z}_{1}}^{2} \nabla_{\vec{z}_{2}}^{2} \\
& +\left[\rho_{1} D_{2}^{(C)} K_{12} \cdot\left(\vec{z}_{1} \nabla_{\vec{z}_{1}}^{2}-\lambda_{1} \partial_{\vec{z}_{1}}\right)+\rho_{1} \rho_{2} \lambda_{1} \partial_{\vec{z}_{1}} \cdot\left(\lambda_{2} \partial_{\vec{z}_{2}}-\vec{z}_{2}\right)+(1 \leftrightarrow 2)\right],
\end{aligned}
$$

where

$$
\begin{aligned}
D_{a}^{(C)} & \equiv\left(\vec{k}_{a} \cdot \vec{z}_{a}\right) \nabla_{z_{a}}^{2}-\lambda_{a}\left(\vec{k}_{a} \cdot \partial_{\vec{z}_{a}}\right), \\
\rho_{a} & \equiv d-1-\Delta_{a}+S_{a}, \\
\lambda_{a} & \equiv 2 S_{a}+d-4 .
\end{aligned}
$$

Finally, we need to highlight that, in momentum space, correlation functions take the form

$$
\left\langle O_{\vec{k}_{1}} \cdots O_{\vec{k}_{n}}\right\rangle=(2 \pi)^{d} \delta^{d}\left(\vec{k}_{1}+\cdots+\vec{k}_{n}\right)\left\langle O_{\vec{k}_{1}} \cdots O_{\vec{k}_{n}}\right\rangle^{\prime} .
$$


We often want to act with weight-shifting operators directly on the primed correlator, with the delta function removed. In that case, one might be concerned that the momentum space operators would also act on the delta function, leading to extra terms after integrationby-parts. This situation is familiar from the Fourier space action of the dilation operator. However, inspection of the weight-shifting operators presented in this section reveals that derivatives always appear in the combination $\vec{K}_{12}$ defined in (B.5), so that they depend on momentum differences and will therefore pass through the delta function.

\section{B.3 A few simple examples}

To illustrate the power of these spinning weight-shifting operators, we give a couple simple applications. We will present a much more systematic study of spinning correlation functions in [57].

- As a first example, let us consider spinning up the two-point function of scalar operators to obtain the two-point function of operators with spin. The scalar two-point function is given by

$$
\langle O O\rangle=k^{2 \Delta-d}
$$

where the normalization is arbitrary. We want to act on this object with the spinraising operator (B.10). Momentum conservation implies that $\vec{k}_{1}=-\vec{k}_{2}$, so that the spin-raising operator simplifies to

$$
\begin{aligned}
\mathcal{S}_{12}^{++}= & (1-S-\Delta)^{2} \vec{z}_{1} \cdot \vec{z}_{2}+(1-S-\Delta)\left[\left(\vec{k} \cdot \vec{z}_{1}\right) \vec{z}_{2} \cdot \partial_{\vec{k}}+\left(\vec{k} \cdot \vec{z}_{2}\right) \vec{z}_{1} \cdot \partial_{\vec{k}}\right] \\
& +\frac{\left(\vec{k} \cdot \vec{z}_{1}\right)\left(\vec{k} \cdot \vec{z}_{2}\right)}{2} \partial_{\vec{k}} \cdot \partial_{\vec{k}} .
\end{aligned}
$$

Acting with this operator repeatedly on $\langle O O\rangle$ generates the two-point function of spinning operators

$$
\begin{aligned}
\left\langle O^{(S)} O^{(S)}\right\rangle & =\left(\mathcal{S}^{++}\right)^{S}\langle O O\rangle \\
& \propto \frac{\left[\left(\hat{k} \cdot \vec{z}_{1}\right)\left(\hat{k} \cdot \vec{z}_{2}\right)\right]^{S}}{k^{d-2 \Delta}} P_{S}^{(\Delta-S-d / 2, d / 2-2)}\left(1-\frac{\vec{z}_{1} \cdot \vec{z}_{2}}{\left(\hat{k} \cdot \vec{z}_{1}\right)\left(\hat{k} \cdot \vec{z}_{2}\right)}\right),
\end{aligned}
$$

where $P_{S}^{(a, b)}$ is the Jacobi polynomial.

- As a simple higher-point example, we construct the three-point correlation function between the stress tensor and two $\Delta=2$ scalars in $d=3$ dimensions from a scalar seed. It is well-known that this correlation function is completely fixed by conformal invariance, and here we indeed reproduce this result. We start with the three-point function of $\Delta=3$ scalar fields:

$$
\langle\phi \phi \phi\rangle=\log \left(k_{t} / \mu\right) \sum_{a} k_{a}^{3}-\sum_{a \neq b} k_{a}^{2} k_{b}+k_{1} k_{2} k_{3},
$$

where $k_{t} \equiv k_{1}+k_{2}+k_{3}$. By applying the $D_{12}$ operator we can lower the weights of two of the scalars to $\Delta=2$, while spinning up the third one to $S=2$. Explicitly, we 
find

$$
\begin{aligned}
\langle T \varphi \varphi\rangle= & D_{13} D_{12}\langle\phi \phi \phi\rangle \\
= & \frac{9\left(k_{1}-k_{2}+k_{3}\right)^{2}}{2 k_{1} k_{t}^{2}}\left(\vec{k}_{1} \cdot \vec{z}_{1}\right)^{2}+\frac{36\left(k_{1}+k_{3}\right)}{k_{t}^{2}}\left(\vec{k}_{1} \cdot \vec{z}_{1}\right)\left(\vec{k}_{2} \cdot \vec{z}_{1}\right) \\
& +\frac{18\left(2 k_{1}+k_{2}+k_{3}\right)}{k_{t}^{2}}\left(\vec{k}_{2} \cdot \vec{z}_{1}\right)^{2} .
\end{aligned}
$$

Since the correlation function of any spinning operator with two scalars is uniquely fixed by conformal invariance, this answer is guaranteed to be conserved, because the spin-2 operator has $\Delta=3$. It can be checked that this agrees with the results of [22], who derived this correlation function in momentum space by directly solving the conformal Ward identity differential equations. Finally, we can use the weightraising operator $\mathcal{W}_{23}^{++}$to raise the scalar weights to $\Delta=3$, which also yields a result that agrees with $[15,22]$.

\section{Polarization tensors}

Our derivation of the spin-exchange solutions to the conformal Ward identities in section 4.2 required explicit expressions for the polarization tensors of spinning operators in a conformal field theory. Fortunately, these can be obtained from the two-point functions of spinning operators (B.19) in a relatively straightforward way. In this appendix, we describe this construction and provide explicit formulas for the relevant polarization sums.

We being by considering the two-point function of spin- $S$ operators

$$
\left\langle O^{(S)} O^{(S)}\right\rangle=\frac{(-2)^{S} S !}{(\tilde{\Delta}-1)_{S}} \frac{\left[\left(\hat{k} \cdot \vec{z}_{1}\right)\left(\hat{k} \cdot \vec{z}_{2}\right)\right]^{S}}{k^{d-2 \tilde{\Delta}}} P_{S}^{(\tilde{\Delta}-S-d / 2, d / 2-2)}(\omega),
$$

where the weight appearing, $\tilde{\Delta}=d-\Delta$, is the shadow dimension to that of the exchanged operator - because we are interested in the inverse of the two-point function - and the argument of the Jacobi polynomial is

$$
\omega \equiv 1-\frac{\vec{z}_{1} \cdot \vec{z}_{2}}{\left(\hat{k} \cdot \vec{z}_{1}\right)\left(\hat{k} \cdot \vec{z}_{2}\right)}
$$

The polarization tensor used in the main text is then defined as

$$
\left(\Pi_{S}\right)_{j_{1} \cdots j_{S}}^{i_{1} \cdots i_{S}}=\frac{1}{\left(S !\left(\frac{d-2}{2}\right)_{S}\right)^{2}} D_{z_{1}}^{i_{1}} \cdots D_{z_{1}}^{i_{S}} D_{j_{1}}^{z_{2}} \cdots D_{j_{S}}^{z_{2}}\left(k^{d-2 \tilde{\Delta}}\left\langle O^{(S)} O^{(S)}\right\rangle\right)
$$

where $D_{z}^{i}$ is the Todorov operator that strips off the null vectors $z^{i}$ from the index-free form of the two-point function:

$$
D_{z}^{i}=\left(\frac{d}{2}-1+\vec{z} \cdot \frac{\partial}{\partial \vec{z}}\right) \frac{\partial}{\partial z_{i}}-\frac{1}{2} z^{i} \frac{\partial^{2}}{\partial \vec{z} \cdot \partial \vec{z}} .
$$

To see the equivalence of the projector (C.3) with the more familiar expressions, we should work in something closer to the helicity basis. This amounts to decomposing $\Pi_{S}$ into a set of irreducible components. We first show how this works for spins 1 and 2, before turning to the general case. 
- Spin 1: specializing (C.3) to the case of spin one, we get

$$
\left(\Pi_{1}\right)_{j}^{i}=\delta_{j}^{i}-\frac{(d-2 \Delta)}{(d-\Delta-1)} \hat{k}^{i} \hat{k}_{j} .
$$

Introducing the projector

$$
\pi_{j}^{i} \equiv \delta_{j}^{i}-\hat{k}^{i} \hat{k}_{j},
$$

this can be split into transverse and longitudinal components

$$
\left(\Pi_{1}\right)_{j}^{i}=\pi_{j}^{i}+\frac{(1-\Delta)}{(1-d+\Delta)} \hat{k}^{i} \hat{k}_{j}
$$

which is (4.11).

- Spin 2: in the case of spin two, we decompose the projector in the orthonormal basis of projectors for traceless two-index tensors

$$
\begin{aligned}
& \left(\Pi_{2,2}\right)^{i j}{ }_{l m}=\pi_{(l}^{(i} \pi_{k)}^{j)}-\frac{1}{d-1} \pi^{i j} \pi_{l m}, \\
& \left(\Pi_{2,1}\right)^{i j}{ }_{l m}=2 \hat{k}^{(i} \hat{k}_{(l} \pi_{m)}^{j)}, \\
& \left(\Pi_{2,0}\right)^{i j}{ }_{l m}=\frac{d}{d-1}\left(\hat{k}^{i} \hat{k}^{j}-\frac{1}{d} \delta_{i j}\right)\left(k_{l} k_{m}-\frac{1}{d} \delta^{l m}\right) .
\end{aligned}
$$

Using this, the spin-2 version of (C.3) becomes

$$
\left(\Pi_{2}\right)_{l m}^{i j}=\left(\Pi_{2,2}\right)^{i j}{ }_{l m}+\frac{\Delta}{d-\Delta}\left(\Pi_{2,1}\right)^{i j}{ }_{l m}+\frac{\Delta(\Delta-1)}{(d-\Delta)(d-\Delta-1)}\left(\Pi_{2,0}\right)^{i j}{ }_{l m},
$$

which is (4.18).

- Spin $S$ : for general spins, the polarization tensors can be constructed by methods of harmonic analysis [91], but we can get an intuitive understanding of the answer as follows: after normalizing the highest-helicity projector to 1 , for each lower-helicity state there will be a pole at each of the partially-massless weights that projects out that mode. The numerator for each helicity mode is given by the same polynomial with $\Delta \rightarrow d-\Delta$. The unitary/non-unitary states between the partially-massless points fixes the relative signs between adjacent helicity components. The final answer is

$$
\left(\Pi_{S}\right)_{j_{1} \cdots j_{S}}^{i_{1} \cdots i_{S}}=\sum_{m=0}^{S} \frac{(\Delta-1+m)_{S-m}}{(d-\Delta-1+m)_{S-m}}\left(\Pi_{S, m}\right)_{j_{1} \cdots j_{S}}^{i_{1} \cdots i_{S}}
$$

The polarization tensor contracted with null vectors $\vec{z}_{1}$ and $\vec{z}_{2}$ takes the form [91]

$$
\begin{aligned}
\Pi_{S, m}\left(\vec{z}_{1}, \vec{z}_{2}\right) & \equiv z_{1}^{i_{1}} \cdots z_{1}^{i_{S}}\left(\Pi_{S, m}\right)_{j_{1} \cdots j_{S}}^{i_{1} \cdots i_{S}} z_{2}^{j_{1}} \cdots z_{2}^{j_{S}} \\
& =\frac{(d-3+2 m)(-1)^{m} S !}{(S-m) !} \frac{\left(\frac{d-2}{2}\right)_{S}}{(d-3)_{S+m+1}}\left[2\left(\hat{k} \cdot \vec{z}_{1}\right)\left(\hat{k} \cdot \vec{z}_{2}\right)\right]^{S} C_{m}^{\frac{d-3}{2}}(\omega)
\end{aligned}
$$


where $C_{m}^{\frac{d-3}{2}}$ is the Gegenbauer polynomial. The explicit polarization tensors can be obtained by stripping off the auxiliary null vectors with the help of the operator (C.4). These polarization tensors satisfy

$$
\begin{aligned}
& \text { orthonormality : } \quad\left(\Pi_{S, m}\right)_{j_{1} \cdots j_{S}}^{i_{1} \cdots i_{S}}\left(\Pi_{S, m^{\prime}}\right)_{l_{1} \cdots l_{S}}^{j_{1} \cdots j_{S}}=\delta_{m m^{\prime}}\left(\Pi_{S, m}\right)_{l_{1} \cdots l_{S}}^{i_{1} \cdots i_{S}}, \\
& \text { completeness : } \\
& 1=\sum_{m=0}^{S} \Pi_{S, m} \\
& \text { transversality : } \quad 0=\hat{k}^{j_{m}} \cdots \hat{k}^{j_{S}}\left(\Pi_{S, m}\right)_{j_{1} \cdots j_{S}}^{i_{1} \cdots i_{S}} .
\end{aligned}
$$

It is also useful to know the harmonic extension of (C.11), which can be contracted with non-null vectors. Contracting with generic vectors $\vec{w}_{1}$ and $\vec{w}_{2}$ leads to [91]

$$
\Pi_{S, m}\left(\vec{w}_{1}, \vec{w}_{2}\right)=2^{S-m} \frac{(m+d / 2-1)_{S-m}}{(2 m+d-2)_{S-m}}\left(\begin{array}{c}
S \\
m
\end{array}\right) L_{S, m}\left(\vec{w}_{1}\right) \Pi_{m, m}\left(\vec{w}_{1}, \vec{w}_{2}\right) L_{S, m}\left(\vec{w}_{2}\right),
$$

where we have defined

$$
\begin{gathered}
L_{S, m}(\vec{w})=2^{m-S}\left(\begin{array}{c}
d / 2+S-1 \\
S-m
\end{array}\right)^{-1}\left(w^{2}\right)^{\frac{S-m}{2}} C_{S-m}^{d / 2+m-1}(\hat{k} \cdot \hat{w}), \\
\Pi_{m, m}\left(\vec{w}_{1}, \vec{w}_{2}\right)=(-2)^{m} m ! \frac{(d / 2-1)_{m}}{(d-3)_{2 m}}\left(\pi^{(11)} \pi^{(22)}\right)^{\frac{m}{2}} C_{m^{\frac{d-3}{2}}}\left(-\frac{\pi^{(12)}}{\sqrt{\pi^{(11)} \pi^{(22)}}}\right) .
\end{gathered}
$$

By design, $L_{S, m}(\vec{w})=(\hat{k} \cdot \vec{w})^{S-m}$ when $w^{2}=0$. The tensors $\pi^{(a b)}$ in $(\mathrm{C} .17)$ correspond to $\pi_{i j}$ of (C.6) contracted with the auxiliary vectors:

$$
\pi^{(a b)}=\vec{w}_{a} \cdot \vec{w}_{b}-\left(\hat{k} \cdot \vec{w}_{a}\right)\left(\hat{k} \cdot \vec{w}_{b}\right) .
$$

In the $s$-channel exchange studied in section 4 , the polarization tensor was associated to the internal momentum $\vec{s}=\vec{k}_{1}+\vec{k}_{2}$. To obtain the polarization sums used in the main text, we therefore let $\vec{w}_{1} \rightarrow \vec{k}_{1}$ and $\vec{w}_{2} \rightarrow \vec{k}_{2}$, and write the result in terms of $\cos \theta_{i} \equiv \hat{k}_{i} \cdot \hat{s}$ and $\psi$ (the angle between $\hat{k}_{1}$ and $\hat{k}_{3}$ projected on the plane perpendicular to $\hat{s}$ ). To take the limit $d \rightarrow 3$, we make use of the following limits of the Gegenbauer polynomials

$$
\begin{aligned}
\lim _{d \rightarrow 3} C_{S-m}^{d / 2-m-1}(z) & =\frac{2^{1-m}}{\left(\frac{3}{2}\right)_{m-1}} \frac{(-1)^{m}}{\left(1-z^{2}\right)^{m / 2}} P_{S}^{m}(z), \\
\lim _{d \rightarrow 3} \frac{m}{d-3} C_{m^{\frac{d-3}{2}}}(\cos \psi) & =\cos (m \psi) \times\left\{\begin{array}{ll}
1 & m=1,2, \cdots \\
\frac{1}{2} & m=0
\end{array} .\right.
\end{aligned}
$$

This gives

$$
\lim _{d \rightarrow 3} \Pi_{S, m}\left(\hat{k}_{1}, \hat{k}_{3}\right)=\frac{S !}{(2 S-1) ! !}\left(2-\delta_{m 0}\right)(-1)^{m} \cos (m \psi) P_{S}^{m}\left(\cos \theta_{1}\right) P_{S}^{-m}\left(\cos \theta_{3}\right),
$$

which is the polarization sum used in (4.31). 


\section{Notation and conventions}

\begin{tabular}{|c|c|c|}
\hline Symbol & Meaning & Reference \\
\hline$\vec{k}$ & Three-momentum vector & Section 2.1 \\
\hline$k_{i}$ & Spatial component of $\vec{k}$ & Section 2.1 \\
\hline$\vec{k}_{a}$ & Momentum of the $a$-th leg & Section 2.1 \\
\hline$k_{a}$ & Magnitude of $\vec{k}_{a}, k_{a} \equiv\left|\vec{k}_{a}\right|$ & Section 2.1 \\
\hline$\hat{k}_{a}$ & Unit vector, $\hat{k}_{a} \equiv \vec{k}_{a} / k_{a}$ & Section 4.2 \\
\hline$k_{t}$ & Sum of momentum magnitudes, $k_{t} \equiv \sum_{a=1}^{N} k_{a}$ & Section 5.4 \\
\hline$s$ & Exchange momentum, $s \equiv\left|\vec{k}_{1}+\vec{k}_{2}\right|$ & Section 2.1 \\
\hline$t$ & Exchange momentum, $t \equiv\left|\vec{k}_{2}+\vec{k}_{3}\right|$ & Section 2.1 \\
\hline$u$ & Momentum ratio, $u \equiv s /\left(k_{1}+k_{2}\right)$ & Eq. $(2.10)$ \\
\hline$v$ & Momentum ratio, $v \equiv s /\left(k_{3}+k_{4}\right)$ & Eq. $(2.10)$ \\
\hline$\vec{\alpha}$ & Difference of momentum vectors, $\vec{\alpha} \equiv \vec{k}_{1}-\vec{k}_{2}$ & Section 4.2 \\
\hline$\vec{\beta}$ & Difference of momentum vectors, $\vec{\beta} \equiv \vec{k}_{3}-\vec{k}_{4}$ & Section 4.2 \\
\hline$\hat{\alpha}$ & Dimensionless difference of momenta, $\hat{\alpha} \equiv\left(k_{1}-k_{2}\right) / s$ & Eq. (2.17) \\
\hline$\hat{\beta}$ & Dimensionless difference of momenta, $\hat{\beta} \equiv\left(k_{3}-k_{4}\right) / s$ & Eq. $(2.17)$ \\
\hline$\hat{\tau}$ & Angular variable, $\hat{\tau} \equiv \vec{\alpha} \cdot \vec{\beta} / s^{2}$ & Eq. (2.17) \\
\hline$\hat{T}$ & Angular variable, $\hat{T} \equiv \hat{\tau}+\hat{\alpha} \hat{\beta} /(u v)$ & Eq. (4.29) \\
\hline$\hat{L}$ & Angular variable & Eq. $(4.30)$ \\
\hline$\gamma$ & Angle between $\hat{k}_{1}$ and $\hat{k}_{3}, \cos \gamma \equiv \hat{k}_{1} \cdot \hat{k}_{3}$ & Eq. (4.28) \\
\hline$\theta_{a}$ & Angle between $\hat{k}_{a}$ and $\hat{s}, \cos \theta_{a} \equiv \hat{k}_{a} \cdot \hat{s}$ & Eq. (4.28) \\
\hline$\psi$ & Projected angle between $\hat{k}_{1}$ and $\hat{k}_{3}, \cos \psi \equiv \hat{T} / \hat{L}^{2}$ & Eq. (4.28) \\
\hline$\Pi_{S, m}$ & Polarization sum & Eq. (4.27) \\
\hline$X$ & Embedding space coordinate & Section 3.1 \\
\hline$Z$ & Embedding space null vector & Section 3.2 \\
\hline$X^{M}$ & Component of $X$ & Section 3.1 \\
\hline$Z^{M}$ & Component of $Z$ & Section 3.2 \\
\hline$X_{a b}$ & Dot product, $X_{a b} \equiv X_{a} \cdot X_{b}$ & Section 3.2 \\
\hline$U$ & Cross ratio, $U \equiv X_{12} X_{34} / X_{13} X_{24}$ & Section 3.3 \\
\hline$V$ & Cross ratio, $V \equiv X_{14} X_{32} / X_{13} X_{24}$ & Section 3.3 \\
\hline$O^{(S)}$ & Index-free spin- $S$ operator, $O^{(S)} \equiv O_{M_{1} \cdots M_{S}} Z^{M_{1}} \cdots Z^{M_{S}}$ & Section 3.2 \\
\hline$\sigma$ & Generic bulk scalar field & Section 2.1 \\
\hline$\varphi$ & Conformally-coupled scalar field & Section 2.2 \\
\hline$\phi$ & Massless scalar field & Section 2.4 \\
\hline$O$ & Operator dual to $\sigma$ & Section 2.1 \\
\hline
\end{tabular}




\begin{tabular}{|c|c|c|}
\hline Symbol & Meaning & Reference \\
\hline$\Delta$ & Scaling dimension (conformal weight) & Section 2.1 \\
\hline$\varphi$ & Operator dual to $\varphi(\Delta=2)$ & Section 2.2 \\
\hline$\phi$ & Operator dual to $\phi(\Delta=3)$ & Section 2.4 \\
\hline$\Delta_{t}$ & Total conformal weight, $\Delta_{t} \equiv \sum_{n} \Delta_{n}$ & Section 2.1 \\
\hline$M$ & Mass parameter & Section 2.2 \\
\hline$S$ & Spin of exchanged particle & Section 4.2 \\
\hline$m$ & Helicity of exchanged particle & Section 4.2 \\
\hline$T$ & Depth of partially massless field & Section 5.4 \\
\hline$F$ & Scalar four-point function & Eq. (2.6) \\
\hline$\hat{F}$ & Dimensionless four-point function, $\hat{F} \equiv s^{9-\Delta_{t}} F$ & Eq. (2.9) \\
\hline$\hat{C}$ & Contact four-point function & Eq. (2.12) \\
\hline$F^{(S)}$ & Four-point function from spin- $S$ exchange & Eq. (4.25) \\
\hline$F_{L}^{(S)}$ & Longitudinal part of four-point function & Eq. (5.11) \\
\hline$B^{(S)}$ & Bispectrum from spin- $S$ exchange & Eq. (5.16) \\
\hline$B_{c}$ & Contact contributions to the bispectrum & Eq. (5.21) \\
\hline$B_{\text {inf }}$ & Bispectrum of slow-roll inflation & Eq. $(5.22)$ \\
\hline$\epsilon$ & Slow-roll parameter & Section 5.3 \\
\hline$\theta$ & Angle in the squeezed limit & Eq. (5.24) \\
\hline$\Delta_{u}$ & Differential operator, $\Delta_{u} \equiv u^{2}\left(1-u^{2}\right) \partial_{u}^{2}-2 u^{3} \partial_{u}$ & Eq. (2.11) \\
\hline$D_{u v}$ & Differential operator, $D_{u v} \equiv(u v)^{2} \partial_{u} \partial_{v}$ & Eq. (4.12) \\
\hline$\vec{K}_{a b}$ & Vector differential operator, $\vec{K}_{a b} \equiv \partial_{\vec{k}_{a}}-\partial_{\vec{k}_{b}}$ & Eq. (B.5) \\
\hline $\mathcal{W}_{a b}^{++}$ & Weight-raising operator & Eq. (B.6) \\
\hline $\mathcal{W}_{a b}^{--}$ & Weight-lowering operator & Eq. (B.4) \\
\hline $\mathcal{W}_{a b}$ & Weight-raising operator, $\mathcal{W}_{a b} \equiv \mathcal{W}_{a b}^{++}$ & Eq. $(5.5)$ \\
\hline$U_{a b}^{(S, m)}$ & Helicity-decomposed weight-shifting operator & Eq. $(5.8)$ \\
\hline $\mathcal{S}_{a b}^{++}$ & Spin-raising operator & Eq. (B.10) \\
\hline $\mathcal{S}_{a b}^{--}$ & Spin-lowering operator & Eq. (B.14) \\
\hline $\mathcal{S}_{a b}$ & Spin-raising operator, $\mathcal{S}_{a b} \equiv \mathcal{S}_{a b}^{++}$ & Eq. $(4.4)$ \\
\hline $\mathcal{D}_{u v}^{(S, m)}$ & Helicity-decomposed spin-raising operator & Eq. $(4.26)$ \\
\hline$D_{12}$ & Operator that raises spin at 1 and lowers weight at 2 & Eq. $(3.20)$ \\
\hline$D_{11}$ & Operator that raises spin at 1 and lowers weight at 1 & Eq. (3.19) \\
\hline$H_{12}$ & Operator that raises spin and lowers weight at 1 and 2 & Eq. (B.13) \\
\hline
\end{tabular}




\begin{tabular}{|clc|}
\hline Symbol & Meaning & Reference \\
\hline$d$ & Boundary space dimension & Section B.2 \\
$z^{i}$ & Auxiliary null vector, $z^{2}=0$ & Section 3.2 \\
$\left(\Pi_{S}\right)_{j_{1} \cdots j_{S}}^{i_{1} \cdots i_{S}}$ & Polarization tensor & Eq. (C.3) \\
$D_{z}^{i}$ & Todorov operator for $z^{i}$ & Eq. (C.4) \\
$\pi_{i j}$ & Spin-1 projector & Eq. (C.6) \\
$\delta_{a b}$ & Kronecker delta & Ref. [92] \\
$P_{S}$ & Legendre polynomial & Ref. [92] \\
$P_{S}^{m}$ & Associated Legendre polynomial & Ref. [92] \\
$C_{m}^{\lambda}$ & Gegenbauer polynomial & Ref. [92] \\
$P_{S}^{(a, b)}$ & Jacobi polynomial & Ref. [92] \\
$(\cdot)_{n}$ & Pochhammer symbol & Ref. [92] \\
\hline
\end{tabular}

Open Access. This article is distributed under the terms of the Creative Commons Attribution License (CC-BY 4.0), which permits any use, distribution and reproduction in any medium, provided the original author(s) and source are credited.

\section{References}

[1] N. Arkani-Hamed, D. Baumann, H. Lee and G.L. Pimentel, The cosmological bootstrap: inflationary correlators from symmetries and singularities, JHEP 04 (2020) 105 [arXiv: 1811.00024] [INSPIRE].

[2] N. Arkani-Hamed, P. Benincasa and A. Postnikov, Cosmological polytopes and the wavefunction of the universe, arXiv:1709.02813 [INSPIRE].

[3] N. Arkani-Hamed and P. Benincasa, On the emergence of Lorentz invariance and unitarity from the scattering facet of cosmological polytopes, arXiv:1811.01125 [INSPIRE].

[4] P. Benincasa, From the flat-space S-matrix to the wavefunction of the universe, arXiv: 1811.02515 [INSPIRE].

[5] C. Sleight, A Mellin space approach to cosmological correlators, JHEP 01 (2020) 090 [arXiv: 1906.12302] [INSPIRE].

[6] C. Sleight and M. Taronna, Bootstrapping inflationary correlators in Mellin space, JHEP 02 (2020) 098 [arXiv: 1907.01143] [INSPIRE].

[7] P. Benincasa, Cosmological polytopes and the wavefuncton of the universe for light states, arXiv: 1909.02517 [INSPIRE].

[8] J.M. Maldacena and G.L. Pimentel, On graviton non-Gaussianities during inflation, JHEP 09 (2011) 045 [arXiv: 1104.2846] [InSPIRE].

[9] I. Antoniadis, P.O. Mazur and E. Mottola, Conformal invariance, dark energy, and CMB non-Gaussianity, JCAP 09 (2012) 024 [arXiv:1103.4164] [INSPIRE]. 
[10] P. Creminelli, Conformal invariance of scalar perturbations in inflation, Phys. Rev. D 85 (2012) 041302 [arXiv: 1108.0874] [INSPIRE].

[11] A. Kehagias and A. Riotto, Operator product expansion of inflationary correlators and conformal symmetry of de Sitter, Nucl. Phys. B 864 (2012) 492 [arXiv:1205.1523] [INSPIRE].

[12] N. Arkani-Hamed and J. Maldacena, Cosmological collider physics, arXiv:1503.08043 [INSPIRE].

[13] S. Raju, New recursion relations and a flat space limit for AdS/CFT correlators, Phys. Rev. D 85 (2012) 126009 [arXiv:1201.6449] [INSPIRE].

[14] S. Raju, Four point functions of the stress tensor and conserved currents in $A d S_{4} / C F T_{3}$, Phys. Rev. D 85 (2012) 126008 [arXiv:1201.6452] [INSPIRE].

[15] I. Mata, S. Raju and S. Trivedi, CMB from CFT, JHEP 07 (2013) 015 [arXiv:1211.5482] [INSPIRE].

[16] A. Ghosh, N. Kundu, S. Raju and S.P. Trivedi, Conformal invariance and the four point scalar correlator in slow-roll inflation, JHEP 07 (2014) 011 [arXiv:1401.1426] [INSPIRE].

[17] N. Kundu, A. Shukla and S.P. Trivedi, Constraints from conformal symmetry on the three point scalar correlator in inflation, JHEP 04 (2015) 061 [arXiv:1410.2606] [INSPIRE].

[18] N. Kundu, A. Shukla and S.P. Trivedi, Ward identities for scale and special conformal transformations in inflation, JHEP 01 (2016) 046 [arXiv: 1507.06017] [INSPIRE].

[19] C. Corianò, L. Delle Rose, E. Mottola and M. Serino, Solving the conformal constraints for scalar operators in momentum space and the evaluation of Feynman's master integrals, JHEP 07 (2013) 011 [arXiv: 1304.6944] [INSPIRE].

[20] C. Corianò and M.M. Maglio, Exact correlators from conformal Ward identities in momentum space and the perturbative TJJ vertex, Nucl. Phys. B 938 (2019) 440 [arXiv: 1802.07675] [INSPIRE].

[21] C. Corianò and M.M. Maglio, On some hypergeometric solutions of the conformal Ward identities of scalar 4-point functions in momentum space, JHEP 09 (2019) 107 [arXiv: 1903.05047] [INSPIRE].

[22] A. Bzowski, P. McFadden and K. Skenderis, Implications of conformal invariance in momentum space, JHEP 03 (2014) 111 [arXiv:1304.7760] [INSPIRE].

[23] D. Anninos, T. Anous, D.Z. Freedman and G. Konstantinidis, Late-time structure of the Bunch-Davies de Sitter wavefunction, JCAP 11 (2015) 048 [arXiv:1406.5490] [INSPIRE].

[24] A. Bzowski, P. McFadden and K. Skenderis, Scalar 3-point functions in CFT: renormalisation, $\beta$-functions and anomalies, JHEP 03 (2016) 066 [arXiv:1510.08442] [INSPIRE].

[25] A. Bzowski, P. McFadden and K. Skenderis, Evaluation of conformal integrals, JHEP 02 (2016) 068 [arXiv: 1511.02357] [inSPIRE].

[26] A. Bzowski, P. McFadden and K. Skenderis, Renormalised 3-point functions of stress tensors and conserved currents in CFT, JHEP 11 (2018) 153 [arXiv:1711.09105] [INSPIRE].

[27] H. Isono, T. Noumi and G. Shiu, Momentum space approach to crossing symmetric CFT correlators, JHEP 07 (2018) 136 [arXiv:1805.11107] [INSPIRE]. 
[28] H. Isono, T. Noumi and T. Takeuchi, Momentum space conformal three-point functions of conserved currents and a general spinning operator, JHEP 05 (2019) 057 [arXiv: 1903.01110] [INSPIRE].

[29] H. Isono, T. Noumi and G. Shiu, Momentum space approach to crossing symmetric CFT correlators. Part II. General spacetime dimension, JHEP 10 (2019) 183 [arXiv:1908.04572] [INSPIRE].

[30] S. Albayrak and S. Kharel, Towards the higher point holographic momentum space amplitudes, JHEP 02 (2019) 040 [arXiv:1810.12459] [INSPIRE].

[31] S. Albayrak, C. Chowdhury and S. Kharel, New relation for Witten diagrams, JHEP 10 (2019) 274 [arXiv: 1904.10043] [InSPIRE].

[32] S. Albayrak and S. Kharel, Towards the higher point holographic momentum space amplitudes. Part II. Gravitons, JHEP 12 (2019) 135 [arXiv:1908.01835] [INSPIRE].

[33] A. Bzowski, P. McFadden and K. Skenderis, Conformal n-point functions in momentum space, Phys. Rev. Lett. 124 (2020) 131602 [arXiv:1910.10162] [INSPIRE].

[34] E. Pajer, G.L. Pimentel and J.V.S. Van Wijck, The conformal limit of inflation in the era of CMB polarimetry, JCAP 06 (2017) 009 [arXiv: 1609.06993] [INSPIRE].

[35] J.A. Farrow, A.E. Lipstein and P. McFadden, Double copy structure of CFT correlators, JHEP 02 (2019) 130 [arXiv:1812.11129] [INSPIRE].

[36] X. Chen and Y. Wang, Quasi-single field inflation and non-Gaussianities, JCAP 04 (2010) 027 [arXiv:0911.3380] [INSPIRE].

[37] D. Baumann and D. Green, Signatures of supersymmetry from the early universe, Phys. Rev. D 85 (2012) 103520 [arXiv:1109.0292] [INSPIRE].

[38] V. Assassi, D. Baumann and D. Green, On soft limits of inflationary correlation functions, JCAP 11 (2012) 047 [arXiv: 1204.4207] [INSPIRE].

[39] X. Chen and Y. Wang, Quasi-single field inflation with large mass, JCAP 09 (2012) 021 [arXiv: 1205.0160] [INSPIRE].

[40] S. Pi and M. Sasaki, Curvature perturbation spectrum in two-field inflation with a turning trajectory, JCAP 10 (2012) 051 [arXiv: 1205.0161] [INSPIRE].

[41] T. Noumi, M. Yamaguchi and D. Yokoyama, Effective field theory approach to quasi-single field inflation and effects of heavy fields, JHEP 06 (2013) 051 [arXiv:1211.1624] [INSPIRE].

[42] D. Baumann, S. Ferraro, D. Green and K.M. Smith, Stochastic bias from non-Gaussian initial conditions, JCAP 05 (2013) 001 [arXiv: 1209.2173] [INSPIRE].

[43] V. Assassi, D. Baumann, D. Green and L. McAllister, Planck-suppressed operators, JCAP 01 (2014) 033 [arXiv: 1304.5226] [INSPIRE].

[44] J.-O. Gong, S. Pi and M. Sasaki, Equilateral non-Gaussianity from heavy fields, JCAP 11 (2013) 043 [arXiv: 1306.3691] [INSPIRE].

[45] H. Lee, D. Baumann and G.L. Pimentel, Non-Gaussianity as a particle detector, JHEP 12 (2016) 040 [arXiv : 1607.03735] [inSPIRE].

[46] A. Kehagias and A. Riotto, On the inflationary perturbations of massive higher-spin fields, JCAP 07 (2017) 046 [arXiv: 1705.05834] [INSPIRE]. 
[47] S. Kumar and R. Sundrum, Heavy-lifting of gauge theories by cosmic inflation, JHEP 05 (2018) 011 [arXiv : 1711.03988] [INSPIRE].

[48] H. An, M. McAneny, A.K. Ridgway and M.B. Wise, Quasi single field inflation in the non-perturbative regime, JHEP 06 (2018) 105 [arXiv: 1706.09971] [INSPIRE].

[49] H. An, M. McAneny, A.K. Ridgway and M.B. Wise, Non-Gaussian enhancements of galactic halo correlations in quasi-single field inflation, Phys. Rev. D 97 (2018) 123528 [arXiv: 1711.02667] [INSPIRE].

[50] D. Baumann, G. Goon, H. Lee and G.L. Pimentel, Partially massless fields during inflation, JHEP 04 (2018) 140 [arXiv:1712.06624] [INSPIRE].

[51] G. Goon, K. Hinterbichler, A. Joyce and M. Trodden, Shapes of gravity: tensor non-Gaussianity and massive spin-2 fields, JHEP 10 (2019) 182 [arXiv:1812.07571] [INSPIRE].

[52] S. Kumar and R. Sundrum, Seeing higher-dimensional grand unification in primordial non-Gaussianities, JHEP 04 (2019) 120 [arXiv:1811.11200] [INSPIRE].

[53] T. Liu, X. Tong, Y. Wang and Z.-Z. Xianyu, Probing P and CP-violations on the cosmological collider, JHEP 04 (2020) 189 [arXiv:1909.01819] [INSPIRE].

[54] S. Kumar and R. Sundrum, Cosmological collider physics and the curvaton, JHEP 04 (2020) 077 [arXiv: 1908.11378] [INSPIRE].

[55] S. Alexander, S.J. Gates, L. Jenks, K. Koutrolikos and E. McDonough, Higher spin supersymmetry at the cosmological collider: sculpting SUSY rilles in the CMB, JHEP 10 (2019) 156 [arXiv: 1907.05829] [inSPIRE].

[56] S. Kim, T. Noumi, K. Takeuchi and S. Zhou, Heavy spinning particles from signs of primordial non-Gaussianities: beyond the positivity bounds, JHEP 12 (2019) 107 [arXiv: 1906.11840] [INSPIRE].

[57] D. Baumann, C. Duaso Pueyo, A. Joyce, H. Lee and G.L. Pimentel, The cosmological bootstrap: spinning correlators from symmetries and factorization, arXiv:2005.04234 [INSPIRE].

[58] M. Gillioz, X. Lu and M.A. Luty, Scale anomalies, states, and rates in conformal field theory, JHEP 04 (2017) 171 [arXiv:1612.07800] [INSPIRE].

[59] M. Gillioz, X. Lu and M.A. Luty, Graviton scattering and a sum rule for the c anomaly in $4 D$ CFT, JHEP 09 (2018) 025 [arXiv:1801.05807] [INSPIRE].

[60] M. Gillioz, Momentum-space conformal blocks on the light cone, JHEP 10 (2018) 125 [arXiv: 1807.07003] [INSPIRE].

[61] T. Bautista and H. Godazgar, Lorentzian CFT 3-point functions in momentum space, JHEP 01 (2020) 142 [arXiv: 1908.04733] [INSPIRE].

[62] M. Gillioz, Conformal 3-point functions and the Lorentzian OPE in momentum space, Commun. Math. Phys. 379 (2020) 227 [arXiv: 1909.00878] [INSPIRE].

[63] M.S. Costa, J. Penedones, D. Poland and S. Rychkov, Spinning conformal blocks, JHEP 11 (2011) 154 [arXiv:1109.6321] [INSPIRE].

[64] D. Karateev, P. Kravchuk and D. Simmons-Duffin, Weight shifting operators and conformal blocks, JHEP 02 (2018) 081 [arXiv:1706.07813] [INSPIRE]. 
[65] M.S. Costa and T. Hansen, AdS weight shifting operators, JHEP 09 (2018) 040 [arXiv: 1805.01492] [INSPIRE].

[66] P.A.M. Dirac, Wave equations in conformal space, Annals Math. 37 (1936) 429 [InSPIRE].

[67] S. Weinberg, Six-dimensional methods for four-dimensional conformal field theories, Phys. Rev. D 82 (2010) 045031 [arXiv: 1006.3480] [INSPIRE].

[68] M.S. Costa, J. Penedones, D. Poland and S. Rychkov, Spinning conformal correlators, JHEP 11 (2011) 071 [arXiv:1107.3554] [INSPIRE].

[69] S. Rychkov, EPFL lectures on conformal field theory in $D \geq 3$ dimensions, SpringerBriefs Phys. (2016) [arXiv:1601.05000] [INSPIRE].

[70] A.M. Polyakov, Conformal symmetry of critical fluctuations, JETP Lett. 12 (1970) 381 [Pisma Zh. Eksp. Teor. Fiz. 12 (1970) 538] [InSPIRE].

[71] P. Kravchuk and D. Simmons-Duffin, Counting conformal correlators, JHEP 02 (2018) 096 [arXiv: 1612.08987] [INSPIRE].

[72] D. Anninos, F. Denef, R. Monten and Z. Sun, Higher spin de Sitter Hilbert space, JHEP 10 (2019) 071 [arXiv: 1711.10037] [inSPIRE].

[73] P. Creminelli, On non-Gaussianities in single-field inflation, JCAP 10 (2003) 003 [astro-ph/0306122] [INSPIRE].

[74] S. Deser and R.I. Nepomechie, Gauge invariance versus masslessness in de Sitter space, Annals Phys. 154 (1984) 396 [INSPIRE].

[75] L. Brink, R.R. Metsaev and M.A. Vasiliev, How massless are massless fields in AdS $S_{d}$, Nucl. Phys. B 586 (2000) 183 [hep-th/0005136] [INSPIRE].

[76] S. Deser and A. Waldron, Partial masslessness of higher spins in (A)dS, Nucl. Phys. B 607 (2001) 577 [hep-th/0103198] [INSPIRE].

[77] S. Deser and A. Waldron, Arbitrary spin representations in de Sitter from dS/CFT with applications to dS supergravity, Nucl. Phys. B 662 (2003) 379 [hep-th/0301068] [INSPIRE].

[78] S. Deser, M. Sandora and A. Waldron, Nonlinear partially massless from massive gravity?, Phys. Rev. D 87 (2013) 101501 [arXiv:1301.5621] [INSPIRE].

[79] C. de Rham, K. Hinterbichler, R.A. Rosen and A.J. Tolley, Evidence for and obstructions to nonlinear partially massless gravity, Phys. Rev. D 88 (2013) 024003 [arXiv:1302.0025] [INSPIRE].

[80] J.M. Maldacena, Non-Gaussian features of primordial fluctuations in single field inflationary models, JHEP 05 (2003) 013 [astro-ph/0210603] [INSPIRE].

[81] L. Dolan, C.R. Nappi and E. Witten, Conformal operators for partially massless states, JHEP 10 (2001) 016 [hep-th/0109096] [INSPIRE].

[82] C. Brust and K. Hinterbichler, Free $\square^{k}$ scalar conformal field theory, JHEP 02 (2017) 066 [arXiv: 1607.07439] [INSPIRE].

[83] C. Brust and K. Hinterbichler, Partially massless higher-spin theory, JHEP 02 (2017) 086 [arXiv: 1610.08510$]$ [INSPIRE].

[84] E. Joung, K. Mkrtchyan and G. Poghosyan, Looking for partially-massless gravity, JHEP 07 (2019) 116 [arXiv: 1904.05915] [inSPIRE]. 
[85] N. Boulanger, C. Deffayet, S. Garcia-Saenz and L. Traina, Theory for multiple partially massless spin-2 fields, Phys. Rev. D 100 (2019) 101701 [arXiv: 1906.03868] [InSPIRE].

[86] P. Benincasa and F. Cachazo, Consistency conditions on the S-matrix of massless particles, arXiv:0705.4305 [INSPIRE].

[87] D.A. McGady and L. Rodina, Higher-spin massless S-matrices in four-dimensions, Phys. Rev. D 90 (2014) 084048 [arXiv:1311.2938] [INSPIRE].

[88] D. Simmons-Duffin, The conformal bootstrap, in Theoretical Advanced Study Institute in Elementary Particle Physics: new frontiers in fields and strings, World Scientific, Singapore (2017), pg. 1 [arXiv: 1602.07982] [INSPIRE].

[89] R.S. Erramilli, L.V. Iliesiu and P. Kravchuk, Recursion relation for general $3 d$ blocks, JHEP 12 (2019) 116 [arXiv: 1907.11247] [InSPIRE].

[90] J. Penedones, E. Trevisani and M. Yamazaki, Recursion relations for conformal blocks, JHEP 09 (2016) 070 [arXiv: 1509.00428] [INSPIRE].

[91] V. Dobrev, G. Mack, V. Petkova, S. Petrova and I. Todorov, Harmonic analysis on the $n$-dimensional Lorentz group and its application to conformal quantum field theory, Lect. Notes Phys. 63 (1977) 1.

[92] Wolfram MathWorld webpage, http://mathworld.wolfram.com/. 